\title{
Using monomer vibrational wavefunctions as contracted basis functions to compute rovibrational levels of an $\mathrm{H}_{2} \mathrm{O}$-atom complex in full dimensionality
}

\author{
Xiao-Gang Wang* and Tucker Carrington Jr. ${ }^{\dagger}$ \\ Chemistry Department, Queen's University, \\ Kingston, Ontario KYL 3N6, Canada
}

(Dated: Nov 14, 2016)

\begin{abstract}
In this paper, we present new ideas for computing rovibrational energy levels of molecules composed of two components and apply them to $\mathrm{H}_{2} \mathrm{O}_{-} \mathrm{Cl}^{-}$. When both components are themselves molecules, euler angles that specify their orientation with respect to an axis system attached to the inter-monomer vector are used as vibrational coordinates. For $\mathrm{H}_{2} \mathrm{O}-\mathrm{Cl}^{-}$, there is only one set of Euler angles. Using Euler angles as intermolecular vibrational coordinates is advantageous because in many cases coupling between them and coordinates that describe the shape of the monomers is unimportant. The monomers are not assumed to be rigid. In the most efficient calculation, vibrational wavefunctions of the monomers are used as contracted basis functions. Energy levels are calculated using the Lanczos algorithm.
\end{abstract}

*Electronic address: xgwang.dalian@gmail.com

${ }^{\dagger}$ Electronic address: Tucker.Carrington@queensu.ca, Fax:613-533-6669 


\section{INTRODUCTION}

Experimentalists and theorists have studied the rovibrational spectroscopy of Van der Waals (VDW) molecules for decades. [1-4] Atom-diatomic dimers were studied first, but in recent years both experimentalists and theorists have published papers about atompolyatomic and polyatomic-polyatomic dimers. [5-27] The large-amplitude radial and angular motion of VDW molecules makes it possible to extract important information about large parts of the potential energy surface (PES). Radial motion corresponds to relative translation of the monomers that are loosely bound to form the VDW molecule. Angular motion corresponds to rotation of the monomers. In this paper we propose new methods for computing the rovibrational spectrum of a VDW dimer that include all of the intramolecular coordinates of the monomers.

To extract information about the PES, it is necessary to have a high-resolution spectrum and a good technique for numerically solving the rovibrational Schroedinger equation. Solving the Schroedinger equation is hard because there are many coordinates. To reduce the cost of calculations, one frequently fixes the values of coordinates that describe the shape of the monomers. Because the intramolecular frequencies are high, compared to the intermolecular frequencies, this is, for many VDW molecules, a good approximation. However, making the monomers rigid clearly makes it impossible to determine how VDW bonds shift monomer energy levels and change the lengths of monomer bonds (e.g. in the water dimer the two $\mathrm{OH}$ bonds of $\mathrm{H}_{2} \mathrm{O}$ are not equally long). In principle, it is clear how to calculate rovibrational levels without fixing the shape of the monomers. The kinetic energy operator (KEO) in polyspherical coordinates is well known. [28, 29] It is easy to write down an exact KEO, valid for any molecule containing $N$ atoms and when it is written in terms of angular momentum operators it is not complicated. [30] It is also known that products of spherical harmonics for each of the $\theta, \phi$ angle pairs are basis functions that enable one to compute wavefunctions that might have amplitude anywhere in configuration space and cope with singularities in the KEO. For radial coordinates, one often uses discrete variable representation (DVR) functions [31] and for rotation Wigner $D_{M, K}^{J}$ functions [32]. Knowing the KEO and having a basis, it is straightforward to compute a spectrum if 1) a PES is available, and 2) it is possible to compute eigenvalues and eigenvectors of the Hamiltonian matrix. 
Computing eigenvalues and eigenvectors of the Hamiltonian matrix is difficult because it is huge. Using an iterative eigensolver, theoretical chemists can nonetheless easily manage bases with millions of functions. [33-41] The basis is large, not only because of the number of coordinates, but also because each basis function is a product of functions of a single variable.

In this paper we report results of calculations of rovibrational energy levels for $\mathrm{H}_{2} \mathrm{O}$ $\mathrm{Cl}^{-}$that include all the coordinates. We compare the approach of the previous paragraph (denoted SH because the angular basis includes products of spherical harmonics) with two new methods. Both of the new methods use the KEO suggested decades ago by Brocks, van der Avoird, Sutcliffe and Tennyson (BAST). [42] The first, like SH, uses a product basis whose functions are products of functions of a single variable, but not polyspherical coordinates; the second uses contracted basis functions that depend on several variables. Using the contracted basis decreases the number of required basis functions by a factor of about 20. Even when using a product basis, the number of basis functions can be reduced by choosing coordinates that are weakly coupled. [43-45]. Although it is possible to compute rovibrational levels of $\mathrm{H}_{2} \mathrm{O}-\mathrm{Cl}^{-}$with the $\mathrm{SH}$ method, the simple polyspherical coordinates are strongly coupled. The coordinates of BAST are better. KEOs similar to that of BAST have been proposed by others. The BAST KEO for a dimer is built from KEOs of two monomers. Gatti and co-workers have also derived the KEO of a molecule composed of two monomers using a frame (called DF frame in this paper and called $E_{2}$ frame by Gatti et al.) specified by only two Euler angles. [43, 46] A similar KEO for a molecule composed of more than two monomers and using a frame specified by three Euler angles was derived by Wang and Carrington. [47] Gatti and co-workers have also derived and used KEOs for molecules composed of what they call sub-systems. [29, 48] The KEOs are complicated when they are not written in terms of angular momentum operators. [48] Some of these ideas have has been reviewed in Ref. 49. BAST were the first to present a KEO for two monomers (subsystems).

In their seminal paper, BAST derived the exact KEO for a molecule consisting of two monomers by using a two-angle frame (called dimer-fixed (DF) frame in this paper) to describe the overall rotation of the molecule and monomer-fixed (MF) frames for each of the monomers. The distance between the centres of mass of the monomers is one intermolecular coordinate. The others are the Euler angles that specify the orientation of the MF frames in 
the DF frame. The intramolecular vibration of the monomers is described by monomer shape coordinates. BAST also proposed using products of Wigner functions as basis functions. Gatti et al. used the BAST approach to compute vibrational energy levels of $\mathrm{NH}_{3}$ by treating it as a dimer composed of a $\mathrm{H}_{3}$ "monomer" and a $\mathrm{N}$ atom. They used Wigner basis functions for the rotation of $\mathrm{H}_{3}$, but did not include the overall rotation. This multi-frame approach was used by Leforestier and co-workers for $\left(\mathrm{H}_{2} \mathrm{O}\right)_{2}[44,45]$. Because of the high dimension of the problem (12D), they used an adiabatic approximation and some of the BAST KEO terms were neglected. In this paper, we use the full BAST KEO.

\section{KINETIC ENERGY OPERATOR}

The coordinates we use are: $(\alpha, \beta)$, that specify the orientation of the $z$ axis of a dimerfixed (DF) frame, which is along the inter-monomer vector $\mathbf{r}_{\mathbf{0}}$, with respect to a space-fixed (SF) frame; three Euler angles, $\left(\alpha^{A}, \beta^{A}, \gamma^{A}\right)$, that specify the orientation of a monomerfixed (MF) frame attached to $\mathrm{H}_{2} \mathrm{O}$, which we denote monomer A (for $\mathrm{H}_{2} \mathrm{O}-\mathrm{Cl}^{-}$the second monomer is an atom, but if this were not the case there would also be a MF frame for the second monomer, monomer B), with respect to the DF frame; the distance $r_{0}$ between the centre of mass of monomer $\mathrm{A}$ and $\mathrm{Cl}$; and $\boldsymbol{q}_{A}$, the three vibrational coordinates that describe the shape of $\mathrm{H}_{2} \mathrm{O}$. We present equations using two different $\boldsymbol{q}_{A}$ : Jacobi coordinates $\boldsymbol{q}_{A}=\left(R_{A}, r_{A}, \theta_{A}\right)$ and Radau coordinates $\boldsymbol{q}_{A}=\left(R_{1 A}, R_{2 A}, \theta_{A}\right)$.

The KEO in these coordinates (atomic units are used in this paper) can be written in compact form by using angular momentum operators in the MF and DF frames[42],

$$
\begin{gathered}
T=T^{A}\left(\mathbf{j}^{\mathrm{MF}, \mathbf{A}}, \boldsymbol{q}_{A}\right)+T_{\text {int }} \\
T_{\text {int }}=-\frac{1}{2 \mu_{0} r_{0}^{2}} \frac{\partial}{\partial r_{0}} r_{0}^{2} \frac{\partial}{\partial r_{0}}+B_{r_{0}}\left(r_{0}\right)\left[J^{2}-\cot \beta \frac{\partial}{\partial \beta}+j^{2}-2 \mathbf{j} \cdot \mathbf{J}\right]
\end{gathered}
$$

with $B_{r_{0}}\left(r_{0}\right)=1 /\left(2 \mu_{r_{0}} r_{0}^{2}\right) \cdot \mu_{r_{0}}$ is the reduced mass of the dimer. $\mathbf{j}=\mathbf{j}^{A}$ and $\mathbf{j}^{\mathrm{MF}, \mathbf{A}}$ are angular momentum operators for $\mathrm{H}_{2} \mathrm{O}$; the components of $\mathbf{j}$ are in the DF frame and components of $\mathbf{j}^{\mathrm{MF}, \mathbf{A}}$ are in the MF frame. If monomer $\mathrm{B}$ is not an atom then $\mathbf{j}=\mathbf{j}^{A}+\mathbf{j}^{B}$. $\mathbf{J}$ is the total angular momentum. Its components in $T_{\text {int }}$ are in the DF frame. The equations for the components of $\mathbf{j}$ are the standard SF angular momentum equations [32] in terms of 
$\alpha^{A}, \beta^{A}, \gamma^{A}$ because the DF frame plays the role of a SF frame for the monomer (it is not embedded in the monomer). The components of $\mathbf{J}$ have a special form which is derived by using the chain rule to change from the three SF Cartesian coordinates of $\boldsymbol{r}_{\mathbf{0}}$ to three new variables $\left(r_{0}, \alpha, \beta\right)[42]$,

$$
\begin{aligned}
& J_{x}=i \frac{1}{\sin \beta} \frac{\partial}{\partial \alpha}+\cot \beta j_{z} \\
& J_{y}=-i \frac{\partial}{\partial \beta} \\
& J_{z}=j_{z} .
\end{aligned}
$$

From Eq. (3) one obtains

$$
\begin{aligned}
J^{2}-\cot \beta \frac{\partial}{\partial \beta} & =-\frac{1}{\sin \beta} \frac{\partial}{\partial \beta} \sin \beta \frac{\partial}{\partial \beta} \\
& +\frac{1}{\sin ^{2} \beta}\left(-\frac{\partial^{2}}{\partial \alpha^{2}}+j_{z}^{2}+2 i \cos \beta \frac{\partial}{\partial \alpha} j_{z}\right)
\end{aligned}
$$

and

$$
J_{ \pm}=J_{x} \pm i J_{y}=i \frac{1}{\sin \beta} \frac{\partial}{\partial \alpha} \pm \frac{\partial}{\partial \beta}+\cot \beta j_{z} .
$$

When monomer $\mathrm{B}$ is not an atom, to specify the position of all atoms in the complex one requires $N_{A}+N_{B}$ vectors. $N_{A}$ and $N_{B}$ are the numbers of atoms in the monomers. One vector is the centre of mass of the VDW molecule and another is $\boldsymbol{r}_{\mathbf{0}}$. The remaining $N_{A}+N_{B}-2$ specify the shape and orientation of the monomers. $j_{z}$ in Eq. (3) is the sum of the $z$ components, in the DF frame, of the angular momenta of these vectors,

$$
j_{z}=-i \sum_{F=A, B} \sum_{i=1}^{N_{F}-1}\left(z_{i, x}^{F} \partial / \partial z_{i, y}^{F}-z_{i, y}^{F} \partial / \partial z_{i, x}^{F}\right),
$$

where $\mathbf{z}_{i}^{F}$ is the DF $z$ component of vector $i$ for monomer $F$, with $F=A, B$, following the original notation of BAST. At this stage, the KEO of monomer $F$ is expressed in terms of Cartesian coordinates in the DF frame:

$$
T^{F}=-\frac{1}{2} \sum_{i, j=1}^{N_{F}-1} \mu_{i j}^{F} \boldsymbol{\nabla} \boldsymbol{z}_{i}^{F} \cdot \nabla \boldsymbol{z}_{j}^{F}
$$

where $\mu_{i j}^{F}$ is a mass factor that depends on the choice of the vectors $\mathbf{z}_{i}^{F}$ (See e.g. Eq. 9 of Ref. 47$). \mu_{i j}^{F}$ is called $G_{i j}^{F}$ in the BAST paper[42]. In the next step, one transforms $T^{F}$ by 
replacing the Cartesian coordinates with rotational $\left(\alpha_{F}, \beta_{F}, \gamma_{F}\right)$ and vibrational coordinates, as is done when deriving a standard KEO for an isolated monomer. It is then convenient to use Wigner functions $\left|j_{F} k_{F} m_{F}\right\rangle$ to represent the rotation of monomer $F$. When using $T_{i n t}$, one does not use the Cartesian expression for $j_{z}$ of Eq. (6) because in the rotational coordinates it is easy to see that $j_{z}$ is diagonal in the Wigner basis. $j_{z}$ behaves as a SF operator when it acts on $\left|j_{F} k_{F} m_{F}\right\rangle$ and as a result a diagonal element of $j_{z}$ is $m_{F}$. One can think of the angles $\alpha, \beta, \alpha_{A}$ as the standard three Euler angles that specify the orientation of a $\mathrm{BF}$ frame embedded whose $\mathrm{z}$-axis is along $\mathbf{r}_{\mathbf{0}}$ and whose $\mathrm{x}$-axis is in the plane containing $\mathbf{r}_{\mathbf{0}}$ and the $\mathrm{MF} z$ axis of $\mathrm{H}_{2} \mathrm{O}$. However, the KEO one obtains when using three Euler angles to embed the frame is less convenient. [42] The potential energy only depends on $\left(\boldsymbol{q}_{A}, \beta_{A}, \gamma_{A}, r_{0}\right)$, which are the 6 vibrational coordinates.

To complete the specification of the KEO of Eq. (1) one must choose $T^{A}\left(\mathbf{j}^{\mathrm{MF}, \mathbf{A}}, \boldsymbol{q}_{A}\right)$. For $T^{A}\left(\mathbf{j}^{\mathrm{MF}, \mathbf{A}}, \boldsymbol{q}_{A}\right)$ one can simply copy and paste any rovibrational KEO for the free monomer. The body-fixed angular momentum components in the rovibrational KEO for the free monomer, which one copies, must be replaced with $\mathbf{j}^{\mathrm{MF}, \mathbf{A}}$ that are projected onto the MF frame, when one pastes. Standard body-fixed (BF) angular momentum operator expressions[32] are used. In $T^{A}\left(\mathbf{j}^{\mathrm{MF}, \mathbf{A}}, \boldsymbol{q}_{A}\right)$, the angular momentum operators depend on $\alpha^{A}, \beta^{A}, \gamma^{A}$. The full expression for $T^{A}$ depends on how the MF frame is attached to the monomer, which will be discussed in section IV.

\section{WIGNER BASIS FUNCTIONS FOR THE RIGID MONOMER CASE AND KEO MATRIX ELEMENTS}

In this section we present the basis functions and KEO matrix elements used for the rigid monomer part of the problem. The $r_{0}$ basis functions are DVR functions. [31] In this section we focus on the angular basis functions. The uncoupled rovibrational angular basis is,

$$
|\xi\rangle=\left|j_{A} k_{A}\left(m_{A}\right) ; J K M\right\rangle
$$

where

$$
\begin{aligned}
\left\langle\alpha_{A}, \beta_{A}, \gamma_{A} \mid j_{A} k_{A} m_{A}\right\rangle & =\sqrt{\frac{2 j_{A}+1}{8 \pi^{2}}} D_{m_{A} k_{A}}^{j_{A}}\left(\alpha_{A}, \beta_{A}, \gamma_{A}\right)^{*} \\
\langle\alpha, \beta, 0 \mid J K M\rangle & =\sqrt{\frac{2 J+1}{4 \pi}} D_{M K}^{J}(\alpha, \beta, 0)^{*},
\end{aligned}
$$


with $D_{M K}^{J}$ being a Wigner function. The normalization factors are different because in the second equation the third Euler angle to zero. We further set $M=0$ and remove $\alpha$. After doing so the angular basis functions are

$$
|\xi\rangle=\left(\frac{1}{\sqrt{2 \pi}}\right)^{3} \bar{d}_{m_{A} k_{A}}^{j_{A}}\left(\beta_{A}\right) \bar{d}_{0 K}^{J}(\beta) e^{i\left(m_{A} \alpha_{A}+k_{A} \gamma_{A}\right)}
$$

where $\bar{d}_{m, k}^{j}=\sqrt{(j+1) / 2} d_{m, k}^{j}$ is a normalized Wigner small- $d$ function. To facilitate the calculation of KEO matrix elements, the constraint $K \equiv m_{A}$ is imposed. In Eq. (8) $m_{A}$ is in brackets because although it occurs in Eq. (8). it is not an independent label, due to the constraint.

Due to the $K \equiv m_{A}$ constraint, matrix elements of the KEO are simple and none of the integrals are singular. It is not obvious that matrix elements of the peculiar operator of Eq. (4) are simple. Consider first the action of $j_{z}$ on a basis function. Because of the constraint,

$$
j_{z}|\xi\rangle=j_{z}^{A}|\xi\rangle=m_{A}|\xi\rangle=K|\xi\rangle
$$

the eigenvalue of $\mathbf{j}^{\mathbf{A}}$ is $m_{A}$ and not $k_{A}$ because $\mathbf{j}^{\mathbf{A}}$ is a $\mathrm{SF}$ angular momentum operator. Starting with Eq. (4) and using Eq. (12), one finds (compare with Eq. 3.41 of Ref. 32),

$$
\begin{aligned}
\left(J^{2}-\cot \beta \frac{\partial}{\partial \beta}\right)|\xi\rangle & =-\frac{1}{\sin \beta} \frac{\partial}{\partial \beta} \sin \beta \frac{\partial}{\partial \beta}+\frac{1}{\sin ^{2} \beta}\left(-\frac{\partial^{2}}{\partial \alpha^{2}}+K^{2}+2 i K \cos \beta \frac{\partial}{\partial \alpha}\right)|\xi\rangle \\
& =J(J+1)|\xi\rangle .
\end{aligned}
$$

Applying the operator in Eq. (13) to $|\xi\rangle$ gives the simple result with the factor of $J(J+1)$ only because of the shared-index basis. Again, because of the constraint, combining Eq. (12) and Eq. (5), one obtains,

$$
J_{ \pm}|\xi\rangle=\lambda_{J K}^{\mp}\left|j_{A} k_{A}\left(m_{A}\right) ; J, K \mp 1, M\right\rangle
$$

with $\lambda_{J K}^{ \pm}=\sqrt{J(J+1)-K(K \pm 1)}$. Therefore, despite the peculiar form of the operators in Eq. (3) and Eq. (4), the operators $J^{2}-\cot \beta \frac{\partial}{\partial \beta}$ and $J_{ \pm}$act on the uncoupled basis of Eq. (8) as if they were standard body-fixed angular momentum operators $J^{2}$ and $J_{ \pm}$, due to the constraint $K \equiv m_{A}$. This was proved by Brocks et al[42] by using the chain rule again to obtain a KEO with a frame whose orientation is specified by three Euler angles. We show that standard-looking matrix elements follow naturally from the constraint. 
The matrix elements of the other operators in $T_{\text {int }}$ can be readily obtained by introducing ladder-operators,

$$
2 \mathbf{j} \cdot \mathbf{J}=j_{+} J_{-}+j_{-} J_{+}+2 j_{z} J_{z}
$$

where $j_{ \pm}=j_{x}^{A} \pm i j_{y}^{A}$. The matrix elements of $T_{i n t}$ are

$$
\begin{aligned}
& \left\langle j_{A}, k_{A},\left(m_{A}\right) ; J K\left|T_{i n t}\right| j_{A}, k_{A},\left(m_{A}\right) ; J K\right\rangle \\
= & B_{r_{0}}\left(r_{0}\right)\left[J(J+1)+j_{A}\left(j_{A}+1\right)-2 K^{2}\right] \\
& \left\langle j_{A}, k_{A},\left(m_{A}+1\right) ; J K+1\left|T_{i n t}\right| j_{A}, k_{A},\left(m_{A}\right) ; J K\right\rangle \\
= & -B_{r_{0}}\left(r_{0}\right) \lambda_{j_{A} m_{A}}^{+} \lambda_{J K}^{+} .
\end{aligned}
$$

The last equation comes from the $j_{+} J_{-}$term. $J_{-}$raises $K$ by 1 because it behaves as a BF operator and $j_{+}$raises $m_{A}$ by 1 because it behaves as a SF operator.

\section{MONOMER KEOS AND MONOMER BASIS FUNCTIONS}

As explained in section II, any triatomic rovibrational KEO can be pasted into Eq. (1). A triatomic rovibrational KEO is determined by choosing the coordinates that describe the shape of the triatomic and choosing how to attach the MF axes to the triatomic. The definition of the axes affects not only the form of the rovibrational KEO of the monomer, but also the meaning of the inter-monomer vibrational angles $\left(\alpha_{A}, \beta_{A}, \gamma_{A}\right)$. Once the coordinates

and axes have been chosen, one selects appropriate monomer basis functions. For all the combinations of shape coordinates, axes and basis considered in this paper, the matrix elements of the free monomer KEO are well known and they can be re-used in a flexible VDW molecule calculation. In this section, we consider three choices for the MF embedding and the shape coordinates: the vector-z embedding with Jacobi coordinates, the bisector embedding with Radau coordinates, and an Eckart embedding with Radau coordinates. In all three cases, the triatomic KEO for monomer $A$ has two parts: the vibrational term $T_{v}^{A}$ and the rovibrational term $T_{v r}^{A}$.

$$
T^{A}=T_{v}^{A}+T_{v r}^{A}
$$




\section{A. Jacobi coordinates and vector-z embedding for MF frame}

The vibrational KEO for $\mathrm{H}_{2} \mathrm{O}$ in the Jacobi coordinates, $R_{A}, r_{A}$ and $\theta_{A}$, where $R_{A}$ is the length of H-H vector $\vec{R}_{A}, r_{A}$ is the length of vector $\vec{r}_{A}$ pointing from the centre of mass of $\mathrm{H}-\mathrm{H}$ to $\mathrm{O}$, and $\theta_{A}$ is the angle between the two vectors, is

$$
\begin{aligned}
T_{v}^{A} & =-\frac{1}{2 \mu_{R_{A}} R_{A}^{2}} \frac{\partial}{\partial R_{A}} R_{A}^{2} \frac{\partial}{\partial R_{A}}-\frac{1}{2 \mu_{r_{A}} r_{A}^{2}} \frac{\partial}{\partial r_{A}} r_{A}^{2} \frac{\partial}{\partial r_{A}}+T_{\theta_{A}} \\
T_{\theta_{A}} & =-\left[B_{R_{A}}\left(R_{A}\right)+B_{r_{A}}\left(r_{A}\right)\right] \frac{1}{\sin \theta_{A}} \frac{\partial}{\partial \theta_{A}} \sin \theta_{A} \frac{\partial}{\partial \theta_{A}},
\end{aligned}
$$

with $B_{R_{A}}\left(R_{A}\right)=1 /\left(2 \mu_{R_{A}} R_{A}^{2}\right)$ and $B_{r_{A}}\left(r_{A}\right)=1 /\left(2 \mu_{r_{A}} r_{A}^{2}\right) . \quad \mu_{R_{A}}$ and $\mu_{r_{A}}$ are appropriate masses. [50] We combine this vibrational part with the rovibrational term for an axis system with the $z$-axis along $\vec{R}_{A}$ and the $y$-axis along $\vec{R}_{A} \times \vec{r}_{A}$. The rovibrational term is

$$
\begin{aligned}
T_{v r}^{A}= & {\left[B_{R_{A}}\left(R_{A}\right)+B_{r_{A}}\left(r_{A}\right)\right] \frac{1}{\sin ^{2} \theta_{A}}\left(j_{z}^{\mathrm{MF}, A}\right)^{2} } \\
& +B_{R_{A}}\left(R_{A}\right)\left[\left(j^{\mathrm{MF}, A}\right)^{2}-2\left(j_{z}^{\mathrm{MF}, A}\right)^{2}\right]-B_{R_{A}}\left(R_{A}\right)\left[j_{-}^{\mathrm{MF}, A} a_{1}^{+}+j_{+}^{\mathrm{MF}, A} a_{1}^{-}\right],
\end{aligned}
$$

with $j_{ \pm}^{\mathrm{MF}, A}=j_{x}^{\mathrm{MF}, A} \pm i j_{y}^{\mathrm{MF}, A}$, and $a_{1}^{ \pm}= \pm \partial / \partial \theta_{A}-\cot \theta_{A} j_{z}^{\mathrm{MF}, A}$. With this $T^{A}$, the KEO (Eq. (1)) is used with the volume element $\left(r_{0}^{2} d r_{0}\right)(\sin \beta d \alpha d \beta)\left(\sin \beta_{A} d \alpha_{A} d \beta_{A} d \gamma_{A}\right)$ $\left(R_{A}^{2} r_{A}^{2} \sin \theta_{A} d R_{A} d r_{A} d \theta_{A}\right)$.

Having specified $T^{A}\left(\mathbf{j}^{\mathrm{MF}, \mathbf{A}}, \boldsymbol{q}_{A}\right)$, it is now possible to choose basis functions for the full problem. Each is a product of the functions in Eq. (8), DVR functions for $r_{A}, r_{0}$, and $R_{A}$ and an associated Legendre function. The angular part is

$$
\left|l_{A},\left(k_{A}\right) ; j_{A}, k_{A},\left(m_{A}\right) ; J K\right\rangle=\Theta_{l_{A}}^{k_{A}}\left|j_{A}, k_{A},\left(m_{A}\right)\right\rangle|J K\rangle .
$$

$\Theta_{l_{A}}^{k_{A}}$ is a normalized associated Legendre function with the Condon-Shortley phase factor. Note that in this basis $k_{A}$ is a shared label. It labels both $\Theta_{l_{A}}^{k_{A}}$ and $\left|j_{A}, k_{A},\left(m_{A}\right)\right\rangle$. We denote this basis, the shared-K (actually $k_{A}$ ) finite basis representation (SKF). This index sharing removes the $1 / \sin ^{2}\left(\theta_{A}\right)$ singularity in $T_{v r}^{A}$. As discussed previously, because $K \equiv m_{A}, m_{A}$ is also shared (between the Wigner function for overall rotation and the Wigner function for rotation of the monomer with respect to the DF frame); this is standard practice for a rigid dimer. In the basis of Eq. (20), $j_{x}^{\mathrm{MF}, A}, j_{y}^{\mathrm{MF}, A}$ and $j_{z}^{\mathrm{MF}, A}$ behave as standard body-fixed angular momentum operators. Therefore, the matrix elements of the monomer rovibrational terms 
are (hereafter dropping the shared $k_{A}$ from the monomer vibrational basis for simplicity)

$$
\begin{aligned}
& \left\langle l_{A} ; j_{A}, k_{A},\left(m_{A}\right) ; J K\left|T_{v r}^{A}\right| l_{A} ; j_{A}, k_{A},\left(m_{A}\right) ; J K\right\rangle \\
= & {\left[B_{R_{A}}\left(R_{A}\right)+B_{r_{A}}\left(r_{A}\right)\right] l_{A}\left(l_{A}+1\right)+B_{R_{A}}\left(R_{A}\right)\left[j_{A}\left(j_{A}+1\right)-2 k_{A}^{2}\right] } \\
& \left\langle l_{A} ; j_{A}, k_{A}+1,\left(m_{A}\right) ; J K\left|T_{v r}^{A}\right| l_{A} ; j_{A}, k_{A},\left(m_{A}\right) ; J K\right\rangle \\
= & -B_{R_{A}}\left(R_{A}\right) \lambda_{l_{A} k_{A}}^{+} \lambda_{j_{A} k_{A}}^{+} .
\end{aligned}
$$

Rovibrational KEOs always have singularities. The monomer rovibrational KEO of Eq. (18) and Eq. (19) is singular when $\theta_{A}=\pi$ and $\theta_{A}=0$. The full KEO of Eq. (1) is singular at $\beta=\pi$ and $\beta=0$. Due to the choice of the Eq. (10) basis functions and the $K \equiv m_{A}$ constraint, despite the $\beta$ singularities, no integrals of $T_{\text {int }}$ are singular. For similar reasons, despite the $\theta_{A}$ singularities, due to the choice of the Eq. (20) basis and the fact that $k_{A}$ is shared, no integrals of $T^{A}\left(\mathbf{j}^{\mathrm{MF}, \mathbf{A}}, \boldsymbol{q}_{A}\right)$ are singular. One might expect that the best possible basis would be one in which all KEO integrals are finite. If this were the case one would use the Jacobi $T^{A}\left(\mathbf{j}^{\mathrm{MF}, \mathbf{A}}, \boldsymbol{q}_{A}\right)$ of this subsection. However, the basis of Eq. (8) is most efficient when the $\mathrm{H}_{2} \mathrm{O}$ monomer is rather rigid and the $\theta_{A}$ singularities are only important when wavefunctions have significant amplitude at or near $\theta_{A}=\pi$ or $\theta_{A}=0$, i.e. when the $\mathrm{H}_{2} \mathrm{O}$ monomer itself undergoes large amplitude motion. Therefore, when the Eq. (8) basis is

good, one expects singularities in $T^{A}\left(\mathbf{j}^{\mathrm{MF}, \mathbf{A}}, \boldsymbol{q}_{A}\right)$ to be unimportant. For the VDW molecule studied in this paper, this is true. The monomer is $\mathrm{H}_{2} \mathrm{O}$ and the potential at $\theta_{A}=0$ is very high and the $\theta_{A}=\pi$ singularity is only important for states with about 5 quanta of bend excitation of $\mathrm{H}_{2} \mathrm{O}$. The Jacobi $T^{A}\left(\mathbf{j}^{\mathrm{MF}, \mathbf{A}}, \boldsymbol{q}_{A}\right)$ might be best for VDW molecules in which the monomers undergo large amplitude motion. In the next two subsections, we present alternative $T^{A}\left(\mathbf{j}^{\mathrm{MF}, \mathbf{A}}, \boldsymbol{q}_{A}\right)$ that are better for $\mathrm{H}_{2} \mathrm{O}-\mathrm{Cl}^{-}$, although some of the matrix elements of the KEO are formally infinite.

\section{B. Radau coordinates and bisector embedding for the MF frame}

Radau coordinates, $R_{1 A}, R_{2 A}$ and $\theta_{A}$, are an alternative to Jacobi coordinates. [51] $R_{i A}$ is the length of Radau vector $\vec{R}_{i A}$ and $\theta_{A}$ is the angle between the two vectors. $T_{v}^{A}$ has the same form as the $T_{v}^{A}$ of the Jacobi case, but the masses are different. When the central atom 
of a triatomic molecule is heavy, as is the case for $\mathrm{H}_{2} \mathrm{O}$, Radau coordinates are similar to bond coordinates. For $\mathrm{H}_{2} \mathrm{O}$, they are a good choice because kinetic coupling is small, there is no stretch-bend cross term, and potential coupling is small, because Radau coordinates are similar to bond coordinates. In this paper we use Radau coordinates with the bisector-z embedding, where the $z$-axis is the negative of the $\theta_{A}$ bisector vector $R_{2 A} \vec{R}_{1 A}+R_{1 A} \vec{R}_{2 A}$ and the $y$-axis is along $\vec{R}_{1 A} \times \vec{R}_{2 A}$. The rovibrational term is

$$
\begin{aligned}
T_{v r}^{A}= & \frac{1}{2}\left[B_{R_{1 A}}\left(R_{1 A}\right)+B_{R_{2 A}}\left(R_{2 A}\right)\right] \\
& \times\left[\frac{1}{1+\cos \theta_{A}}\left(j_{x}^{\mathrm{MF}, A}\right)^{2}+\frac{1}{2}\left(j_{y}^{\mathrm{MF}, A}\right)^{2}+\frac{1}{1-\cos \theta_{A}}\left(j_{z}^{\mathrm{MF}, A}\right)^{2}\right] \\
& +\left[B_{R_{1 A}}\left(R_{1 A}\right)-B_{R_{2 A}}\left(R_{2 A}\right)\right]\left[\frac{1}{2 \sin \theta_{A}}\left\{j_{x}^{\mathrm{MF}, A}, j_{z}^{\mathrm{MF}, A}\right\}-p_{\theta_{A}}^{H} j_{y}^{\mathrm{MF}, A}\right],
\end{aligned}
$$

where

$$
p_{\theta_{A}}^{H}=-i\left(\frac{\partial}{\partial \theta_{A}}+\frac{1}{2} \cot \theta_{A}\right)
$$

is an Hermitian momentum operator and $B_{R_{i A}}\left(R_{i A}\right)=1 /\left(2 \mu_{R_{i A}} R_{i A}^{2}\right)$. With this $T^{A}$, the KEO (Eq. (1)) is used with the volume element $\left(r_{0}^{2} d r_{0}\right)(\sin \beta d \alpha d \beta)\left(\sin \beta_{A} d \alpha_{A} d \beta_{A} d \gamma_{A}\right)$ $\left(R_{1 A}^{2} R_{2 A}^{2} \sin \theta_{A} d R_{1 A} d R_{2 A} d \theta_{A}\right)$. We test two different bases.

1. shared-K finite basis representation (SKF) basis for the monomer

For Radau coordinates KEOs we use an SKF-type basis proposed by Carter, Handy and Sutcliffe[52] that has the same form as Eq. (20). Instead, it would be possible to use the basis

$$
\left|J_{n}^{a, b}\right\rangle\left|j_{A}, k_{A},\left(m_{A}\right)\right\rangle|J K\rangle
$$

where $J_{n}^{a, b}$ is a Jacobi function [53] and $a, b$ depend on $j_{A}$ and $k_{A}$. [54] This basis has the virtue of removing all singularities in bisector-embedded Radau KEOs. In the basis of Eq. (20), diagonal matrix elements with $k_{A}=0$ basis functions of terms $T_{v r}^{A}$ are singular at $\theta_{A}=0$ (due to the $\left(j_{z}^{\mathrm{MF}, A}\right)^{2}$ term) and at $\theta_{A}=\pi$ (due to the $\left(j_{x}^{\mathrm{MF}, A}\right)^{2}$ term). If the $z$ axis is along the bisector, then replacing

$$
\frac{1}{1-\cos \theta_{A}} \rightarrow \frac{2}{\sin ^{2} \theta_{A}}-\frac{1}{1+\cos \theta_{A}}
$$


and exploiting the shared index in Eq. (20), makes it makes it possible, with the SKF basis, to combine $\frac{2}{\sin ^{2} \theta_{A}}\left(j_{z}^{\mathrm{MF}, A}\right)^{2}$ with the bending term $T_{\theta_{A}}$ in $T_{v}^{A}$ of Eq. (18) to obtain $l_{A}\left(l_{A}+1\right)$. The application of the $T_{\theta_{A}}$ term in $T_{v}^{A}$ is then trivial. Thus, the SKF basis removes the singularity of the bisector-z KEO at $\theta_{A}=0$; the singularity at $\theta_{A}=\pi$ remains.

A bisector-x embedding KEO, with the $x$ axis along the bisector is obtained from Eq. (22) by switching the $(x, y, z)$ labels to $(z,-y, x)$. Similar to the bisector-z embedding case, by replacing

$$
\frac{1}{1+\cos \theta_{A}} \rightarrow \frac{2}{\sin ^{2} \theta_{A}}-\frac{1}{1-\cos \theta_{A}}
$$

and combining $\frac{2}{\sin ^{2} \theta_{A}}\left(j_{z}^{\mathrm{MF}, A}\right)^{2}$ with the bending term $T_{\theta_{A}}$, one removes the singularity at $\theta_{A}=\pi$; the singularity at $\theta_{A}=0$ remains. At $\theta_{A}=0$, the potential is very high and the $\theta_{A}=0$ singularity is therefore unimportant. This makes the bisector-x embedding a good choice. At $\theta_{A}=\pi$, the potential may be low enough that the $\theta_{A}=\pi$ singularity is important for highly excited rovibrational levels. This may make the bisector-z embedding less good for high-lying levels, however, both embeddings could be used for the levels we compute because the corresponding wavefunctions have small amplitude at both singularities. We prefer the bisector-z embedding because it allows one to exploit the permutation symmetry of the two $\mathrm{H}$ in $\mathrm{H}_{2} \mathrm{O}$. For the bisector-z embedding, the matrix elements of the monomer rovibrational terms are

$$
\begin{aligned}
& \left\langle l_{A}^{\prime} ; j_{A}, k_{A},\left(m_{A}\right) ; J K\left|T_{v r}^{A}+T_{\theta_{A}}\right| l_{A} ; j_{A}, k_{A},\left(m_{A}\right) ; J K\right\rangle \\
= & {\left[B_{R_{1 A}}\left(R_{1 A}\right)+B_{R_{2 A}}\left(R_{2 A}\right)\right]\left\{\frac{1}{8}\left[j_{A}\left(j_{A}+1\right)-k_{A}^{2}\right] \delta_{l_{A}^{\prime}, l_{A}}+l_{A}\left(l_{A}+1\right) \delta_{l_{A}^{\prime}, l_{A}}\right.} \\
& \left.+\frac{1}{4}\left[j_{A}\left(j_{A}+1\right)-3 k_{A}^{2}\right] E_{l_{A}^{\prime}, l_{A}, k_{A}}\right\} \\
& \left\langle l_{A}^{\prime} ; j_{A}, k_{A}+1,\left(m_{A}\right) ; J K\left|T_{v r}^{A}\right| l_{A} ; j_{A}, k_{A},\left(m_{A}\right) ; J K\right\rangle \\
= & \frac{1}{4}\left[B_{R_{1 A}}\left(R_{1 A}\right)-B_{R_{2 A}}\left(R_{2 A}\right)\right] \lambda_{j_{A} k_{A}}^{+}\left[\left(2 k_{A}+1\right)\left(G_{l_{A}^{\prime}, l_{A}, k_{A}}-D_{l_{A}^{\prime}, l_{A}, k_{A}}\right)-2 \lambda_{l_{A} k_{A}}^{+} \delta_{l_{A}^{\prime} l_{A}}\right] \Omega \\
& \left\langle l_{A}^{\prime} ; j_{A}, k_{A}+2,\left(m_{A}\right) ; J K\left|T_{v r}^{A}\right| l_{A} ; j_{A}, k_{A},\left(m_{A}\right) ; J K\right\rangle \\
= & \frac{1}{16}\left[B_{R_{1 A}}\left(R_{1 A}\right)+B_{R_{2 A}}\left(R_{2 A}\right)\right] \lambda_{j_{A} k_{A}}^{+} \lambda_{j_{A} k_{A}+1}^{+}\left(2 F_{l_{A}^{\prime}, l_{A}, k_{A}}-H_{l_{A}^{\prime}, l_{A}, k_{A}}\right),
\end{aligned}
$$


where $\mathbf{D}, \mathbf{E}, \mathbf{F}, \mathbf{G}$ and $\mathbf{H}$ are matrices representing $\cot \theta_{A}, \frac{1}{1+\cos \theta_{A}}$, and $\frac{1}{\sin \theta_{A}}$. Their elements are computed exactly by Gauss quadrature except for the $k_{A}=0$, elements of $\mathbf{E}$, corresponding to the integral of $\frac{1}{1+\cos \theta_{A}}$, singular at $\theta_{A}=\pi$, which are computed with the same number of Gauss-Legendre points as we use for the potential integrals. See appendix A for detail. The matrix elements of Eq. (27) can be obtained from those previously presented by Sutcliffe and Tennyson[55] for the bisector KEO in bond coordinates (which has more terms than the Radau KEO here) and the SKF basis. We present them here again for completeness and in a different format. Note that Sutcliffe and Tennyson[56] as well as Carter and Handy [52] used non-anomalous commutation relations for the body-fixed angular momentum operators. We use anomalous commutation relations for the body-fixed angular momentum operators, which is the proper thing to do. This changes the sign of the Coriolis term.

For the bisector-x embedding, the matrix elements of the monomer rovibrational terms are

$$
\begin{aligned}
& \left\langle l_{A}^{\prime} ; j_{A}, k_{A},\left(m_{A}\right) ; J K\left|T_{v r}^{A}+T_{\theta_{A}}\right| l_{A} ; j_{A}, k_{A},\left(m_{A}\right) ; J K\right\rangle \\
= & {\left[B_{R_{1 A}}\left(R_{1 A}\right)+B_{R_{2 A}}\left(R_{2 A}\right)\right]\left\{\frac{1}{8}\left[j_{A}\left(j_{A}+1\right)-k_{A}^{2}\right] \delta_{l_{A}^{\prime}, l_{A}}+l_{A}\left(l_{A}+1\right) \delta_{l_{A}^{\prime}, l_{A}}\right.} \\
& \left.+\frac{1}{4}\left[j_{A}\left(j_{A}+1\right)-3 k_{A}^{2}\right] I_{l_{A}^{\prime}, l_{A}, k_{A}}\right\} \\
& \left\langle l_{A}^{\prime} ; j_{A}, k_{A}+1,\left(m_{A}\right) ; J K\left|T_{v r}^{A}\right| l_{A} ; j_{A}, k_{A},\left(m_{A}\right) ; J K\right\rangle \\
= & \frac{1}{4}\left[B_{R_{1 A}}\left(R_{1 A}\right)-B_{R_{2 A}}\left(R_{2 A}\right)\right] \lambda_{j_{A} k_{A}}^{+}\left[\left(2 k_{A}+1\right)\left(G_{l_{A}^{\prime}, l_{A}, k_{A}}+D_{l_{A}^{\prime}, l_{A}, k_{A}}\right)-2 \lambda_{l_{A} k_{A}}^{+} \delta_{l_{A}^{\prime} l_{A}}\right] \\
& \left\langle l_{A}^{\prime} ; j_{A}, k_{A}+2,\left(m_{A}\right) ; J K\left|T_{v r}^{A}\right| l_{A} ; j_{A}, k_{A},\left(m_{A}\right) ; J K\right\rangle \\
= & \frac{1}{16}\left[B_{R_{1 A}}\left(R_{1 A}\right)+B_{R_{2 A}}\left(R_{2 A}\right)\right] \lambda_{j_{A} k_{A}}^{+} \lambda_{j_{A} k_{A}+1}^{+}\left(2 J_{l_{A}^{\prime}, l_{A}, k_{A}}-H_{l_{A}^{\prime}, l_{A}, k_{A}}\right)
\end{aligned}
$$

The matrices $\mathbf{D}, \mathbf{G}, \mathbf{H}, \mathbf{I}$ and $\mathbf{J}$ are given in the appendix. When $k_{A}=0$, matrix elements of $\mathbf{I}$ are singular at $\theta_{A}=0$. 


\section{DVR basis for the monomer}

The SKF basis has the advantage that one of the singularities is treated exactly. However, if wavefunctions have small amplitude at and close to both singularities then that advantage is unimportant. The SKF basis has the disadvantage that, due to the shared indices, it is harder to contract the monomer functions into monomer vibrational eigenfunctions, which

are good basis basis functions for the full problem (section VII). Rather than using $\Theta_{l_{A}}^{k_{A}}$ as $\theta_{A}$ basis functions, one might therefore prefer to use $\Theta_{l_{A}}^{0}$ [ [57]. If this is done, there is no reason not to use the corresponding DVR. [31]

We do calculations with direct product monomer DVR basis functions with the bisector-z embedding KEO. Each function in the complete basis is a product of a Wigner function for the rotation of the VDW molecule, a Wigner function for rotation of $\mathrm{H}_{2} \mathrm{O}$, a DVR function for $r_{0}$, and an $\mathrm{H}_{2} \mathrm{O}$ vibrational basis function. Omitting the $r_{0}$ function, a basis function is

$$
\left|\eta_{1}, \eta_{2}, \eta_{3}\right\rangle\left|j_{A} k_{A}\left(m_{A}\right)\right\rangle|J K\rangle
$$

where $K \equiv m_{A}$, and $\eta_{1}, \eta_{2}$, and $\eta_{3}$ are DVR basis indices for the coordinates $R_{1 A}, R_{2 A}$, and $\theta_{A}$, respectively. Because functions are diagonal in a DVR basis, it is straightforward to derive matrix elements of $T_{v r}^{A}$. Only $p_{\theta_{A}}^{H}$ is not diagonal in the DVR basis and it is discussed in Ref. 57. Matrix elements of the Coriolis term in $T_{v r}^{A}$ are

$$
\begin{aligned}
& \left\langle\eta_{1}, \eta_{2}, \eta_{3}^{\prime} ; j_{A}, k_{A}+1,\left(m_{A}\right) ; J K\left|T_{v r}^{A}\right| \eta_{1}, \eta_{2}, \eta_{3} ; j_{A}, k_{A},\left(m_{A}\right) ; J K\right\rangle \\
= & -\left[B_{R_{1 A}}\left(R_{1 A, \eta_{1}}\right)-B_{R_{2 A}}\left(R_{2 A, \eta_{2}}\right)\right]\left\langle\eta_{3}^{\prime}\left|p_{\theta_{A}}^{H}\right| \eta_{3}\right\rangle \lambda_{j_{A} k_{A}}^{+},
\end{aligned}
$$

\section{Radau coordinates and an Eckart embedding for the MF frame}

We have also used Radau coordinates and an Eckart frame. The Eckart frame has the advantage that it minimizes Coriolis coupling close to the equilibrium geometry. For a bent ABA triatomic molecule, the well known bisector frame is a good alternative to the Eckart frame because at the equilibrium shape the Coriolis term in the bisector KEO is also zero. For other molecules good alternatives to an Eckart frame may not exist. For any triatomic 
monomer, the rovibrational part of the KEO can be written

$$
\begin{aligned}
T_{v r}^{A} & =\frac{1}{2}\left[G_{x, x}\left(j_{x}^{\mathrm{MF}, A}\right)^{2}+G_{y, y}\left(j_{y}^{\mathrm{MF}, A}\right)^{2}+G_{z, z}\left(j_{z}^{\mathrm{MF}, A}\right)^{2}+G_{x, z}\left\{j_{x}^{\mathrm{MF}, A}, j_{z}^{\mathrm{MF}, A}\right\}\right] \\
& +\frac{1}{2} \sum_{k}\left(p_{k}^{H} G_{y, k}+G_{y, k} p_{k}^{H}\right) j_{y}^{\mathrm{MF}, A}
\end{aligned}
$$

where $k$ labels one of the vibrational coordinates. For a triatomic monomer, there is only one non-zero contribution, the one with $k=\theta$, because the Radau coordinates are orthogonal. In the triatomic case, exact equations are known for the rovibrational $G$ matrix elements, $[58,59]$ but it is easier to calculate them at quadrature points numerically. [60] Although it is possible to use a Jacobi basis designed to deal with the singularities of the Eckart KEO, [58] that is unnecessary for the purpose of the calculations of this paper because none of the wavefunctions we compute have significantly amplitude at or close to the singularities. We use the DVR basis of Eq. (29). The Eckart-frame rovibrational $G_{k, y}$ matrix elements are complicated functions of the vibrational coordinates, just like the potential energy function.

In the DVR, however they are diagonal. $p_{k}^{H}$ is not diagonal in the DVR and matrix elements of the Coriolis term are

$$
\begin{aligned}
& \left\langle\eta_{1}, \eta_{2}, \eta_{3}^{\prime} ; j_{A}, k_{A}+1,\left(m_{A}\right) ; J K\left|T_{v r}^{A}\right| \eta_{1}, \eta_{2}, \eta_{3} ; j_{A}, k_{A},\left(m_{A}\right) ; J K\right\rangle \\
= & \frac{1}{2}\left[G_{y, \theta_{A}}\left(\eta_{1}, \eta_{2}, \eta_{3}^{\prime}\right)+G_{y, \theta_{A}}\left(\eta_{1}, \eta_{2}, \eta_{3}\right)\right]\left\langle\eta_{3}^{\prime}\left|p_{\theta_{A}}^{H}\right| \eta_{3}\right\rangle \lambda_{j_{A} k_{A}}^{+} .
\end{aligned}
$$

With the Radau coordinate $T_{v}^{A}$ and the $T_{v r}^{A}$ of Eq. (31) the KEO (Eq. (1)) is used with the volume element $\left(r_{0}^{2} d r_{0}\right)(\sin \beta d \alpha d \beta)\left(\sin \beta_{A} d \alpha_{A} d \beta_{A} d \gamma_{A}\right)\left(R_{A}^{2} r_{A}^{2} \sin \theta_{A} d R_{A} d r_{A} d \theta_{A}\right)$. See Ref. 60 for more details.

\section{USE OF PARITY AND H-H PERMUTATION SYMMETRY OF $\mathrm{H}_{2} \mathrm{O}$}

It is of course advantageous to exploit symmetry. The permutation-inversion group of $\mathrm{Cl}^{-} \mathrm{H}_{2} \mathrm{O}$ contains $E^{*}$ and (12), where (12) permutes the two $\mathrm{H}$ atoms. [61] We use symmetryadapted basis functions and do one Lanczos calculation for each irreducible representation (irrep). A parity adapted (PA) basis can be made by applying $E^{*}$ to the basis functions. The SF Cartesian coordinates of each atom must change sign under $E^{*}$. There are different ways to choose how $E^{*}$ affects the coordinates on which the basis functions depend. The 
DF frame is attached to $\mathbf{r}_{\mathbf{0}}$ and the effect of $E^{*}$ on $\alpha$ and $\beta$ is therefore unambiguous. It is only necessary, for the MF angles and the intra-monomer vibrational coordinates, to make a choice that is consistent with the way $E^{*}$ affects the SF Cartesian coordinates. Of the three possible choices, our preferred choice (Case II of Appendix B) is most convenient. Table I, which gives the effect of the two symmetry operations on the coordinates and basis functions is consistent with this choice. It has the advantage that the corresponding PA basis functions are either real or pure imaginary.

From the results of Table I, it is straightforward to derive rigid-monomer PA basis functions. For the rigid-monomer case, the parity-adapted basis functions are

$$
\begin{aligned}
& u_{j_{A} k_{A}\left(m_{A}\right) ; K}^{J P}=N_{k_{A} K} \frac{1}{\sqrt{2}} \\
& {\left[\left|j_{A} k_{A}\left(m_{A}\right) ; J K\right\rangle+(-1)^{J+k_{A}+P}\left|j_{A} \bar{k}_{A}\left(\bar{m}_{A}\right) ; J \bar{K}\right\rangle\right],}
\end{aligned}
$$

with $N_{k_{A} K}=\left(1+\delta_{k_{A}, 0} \delta_{K, 0}\right)^{-1 / 2}$ where $P=0$ and 1 correspond to even and odd parity, respectively. In the parity-adapted basis, the restrictions on quantum numbers are (i) $K \geq 0$ and (ii) $k_{A} \geq 0$ if $K=0$. Written explicitly,

$$
u_{j_{A} k_{A}\left(m_{A}\right) ; K}^{J P}=N_{k_{A} K} \sqrt{2}\left(\frac{1}{\sqrt{2 \pi}}\right)^{2} \bar{d}_{m_{A} k_{A}}^{j_{A}}\left(\beta_{A}\right) \bar{d}_{0 K}^{J}(\beta) \cos \left(m_{A} \alpha_{A}+k_{A} \gamma_{A}\right)
$$

if $(-1)^{J+P}=1$, and

$$
u_{j_{A} k_{A}\left(m_{A}\right) ; K}^{J P}=i \sqrt{2}\left(\frac{1}{\sqrt{2 \pi}}\right)^{2} \bar{d}_{m_{A} k_{A}}^{j_{A}}\left(\beta_{A}\right) \bar{d}_{0 K}^{J}(\beta) \sin \left(m_{A} \alpha_{A}+k_{A} \gamma_{A}\right)
$$

if $(-1)^{J+P}=-1$.

To make flexible-monomer PA functions from the SKF basis used with either the Jacobi, z-vector embedded KEO or the Radau, z-embedded bisector KEO, one must combine $\left|l_{A} ; j_{A} k_{A}\left(m_{A}\right) ; J K\right\rangle$ and $E^{*}\left|l_{A} ; j_{A} k_{A}\left(m_{A}\right) ; J K\right\rangle$. Using Table I, we find that the flexiblemonomer PA functions are

$$
\begin{aligned}
& u_{l_{A} ; j_{A} k_{A}\left(m_{A}\right) ; K}^{J P}=N_{k_{A} K} \frac{1}{\sqrt{2}} \\
& {\left[\left|l_{A} ; j_{A} k_{A}\left(m_{A}\right) ; J K\right\rangle+(-1)^{J+P}\left|l_{A} ; j_{A} \bar{k}_{A}\left(\bar{m}_{A}\right) ; J \bar{K}\right\rangle\right] .}
\end{aligned}
$$

To calculate matrix-vector products for the KEO in this basis, we transform the Lanczos vector represented in the basis of Eq. (36) to a vector represented in the $\left|l_{A} ; j_{A} k_{A}\left(m_{A}\right) ; J K\right\rangle$ 
basis and then use the ideas of sections III and IV. The transformed vector is obtained by "unpacking" the Lanczos vector in the PA basis into a vector in the non-PA basis, i.e. creating a larger vector by adding components that are equal, with or without a sign change, to those of the Lanczos vector. Increasing the size of the vector is not a problem because although the size of a vector is doubled, it is only necessary to double the size of a bend vector, because the bend terms in the KEO are diagonal in the indices for all three of the stretch coordinates. The flexible-monomer SKF PA functions have the same form in the Jacobi and Radau cases and also in the SH case, when the KEO is written in terms of polyspherical angles. It can be shown that when monomer A has only three atoms, each flexible-monomer PA functions is a product of a monomer vibrational basis function and one of the rigid monomer PA functions of Eq. (33),

$$
u_{l_{A} ; j_{A} k_{A}\left(m_{A}\right) ; K}^{J P}=\Theta_{l_{A}}^{k_{A}}\left(\theta_{A}\right) u_{j_{A} k_{A}\left(m_{A}\right) ; K}^{J P} .
$$

This makes sense because all vibrational wavefunctions of a triatomic molecule have positive parity. When a DVR monomer vibrational basis is used, either with a bisector embedding or with an Eckart embedding, it is actually straightforward to use Eq. (37). In this case, one sets $k_{A}=0$ in $\Theta_{l_{A}}^{k_{A}}$ in Eq. (37). Matrix elements like those of Eq. (30) are computed using the $T$ matrix that relates the $\theta_{A}$ finite basis representation and the discrete variable representation. [31]

The effect of (12) is simpler. Table I lists the effect of (12) on the Jacobi and Radau coordinates, and its effect on the SKF basis functions (used for the Radau/bisector/SKF calculations) and the rigid monomer basis (used with Eq. (37) for the Radau/bisector/DVR and Radau/Eckart/DVR calculations). The last line is because $\Theta_{l_{A}}^{k_{A}}\left(\pi-\theta_{A}\right)=(-1)^{l_{A}+k_{A}}$ $\Theta_{l_{A}}^{k_{A}}\left(\theta_{A}\right)$. From Table I it is clear that each PA function actually transforms like an irrep of of $G_{4}$.

\section{POTENTIAL INTEGRALS IN THE PA BASIS}

In this section, we will present equations for potential matrix elements. The equations of this section enable one to work directly in the PA basis. Leforestier[7] has given equations for potential matrix elements in the basis of Eq. (8) (he studied $\mathrm{H}_{2} \mathrm{O}-\mathrm{Ar}$, assuming $\mathrm{H}_{2} \mathrm{O}$ was rigid). We give equations for potential matrix elements in the PA basis of Eq. (36) in which 
$k_{A}$ is shared between the intra-monomer vibrational basis and the inter-monomer rotational basis and $K=m_{A}$ is shared between the inter-monomer rotational basis and the basis for the overall rotation. Results for the rigid monomer case (Eq. (33) ) can be easily derived as a special case. Leforestier also used a PA basis, however, he transformed (unpacked) Lanczos vectors in the PA basis into vectors in the Eq. (8) basis that are about twice as large and then did the potential integral in the Eq. (8) basis.

Because the potential does not depend on $\beta$ we first integrate out $\beta$. The potential integral is diagonal in $J, P$, and $K$ and $J^{\prime}=J, K^{\prime}=K$ and $P^{\prime}=P$ are applied in the following equations. The full angular part of the potential integral in the SKF basis is

$$
\begin{aligned}
& \left\langle u_{l_{A}^{\prime} ; j_{A}^{\prime} k_{A}^{\prime}\left(m_{A}\right) ; K}^{J P}|V| u_{l_{A} ; j_{A} k_{A}\left(m_{A}\right) ; K}^{J P}\right\rangle= \\
& \int_{0}^{\pi} \sin \theta_{A} d \theta_{A} \int_{0}^{\pi} \sin \beta_{A} d \beta_{A} \Theta_{l_{A}^{\prime}}^{k_{A}^{\prime}}\left(\theta_{A}\right) \Theta_{l_{A}}^{k_{A}}\left(\theta_{A}\right) \bar{d}_{m_{A} k_{A}^{\prime} k_{A}^{\prime}}\left(\beta_{A}\right) \bar{d}_{m_{A} k_{A}}^{j_{A}}\left(\beta_{A}\right) I_{k_{A}^{\prime}, k_{A}, m_{A}}^{d i h}\left(\theta_{A}, \beta_{A}\right)
\end{aligned}
$$

If $(-1)^{J+P}=-1$, then

$$
\begin{aligned}
& I_{k_{A}^{\prime}, k_{A}, m_{A}}^{d i h}\left(\theta_{A}, \beta_{A}\right)= \\
& \int_{0}^{2 \pi} d \alpha_{A} \int_{0}^{2 \pi} d \gamma_{A} \sin \left(m_{A} \alpha_{A}+k_{A}^{\prime} \gamma_{A}\right) V\left(\theta_{A}, \beta_{A}, \gamma_{A}\right) \sin \left(m_{A} \alpha_{A}+k_{A} \gamma_{A}\right) .
\end{aligned}
$$

$I_{k_{A}^{\prime}, k_{A}, m_{A}}^{d i h}$ is derived by substituting Eq. (36) into $\left\langle u_{l_{A}^{\prime} ; j_{A}^{\prime} k_{A}^{\prime}\left(m_{A}\right) ; K}^{J P}|V| u_{l_{A} ; j_{A} k_{A}\left(m_{A}\right) ; K}^{J P}\right\rangle$. A similar equation for $I_{k_{A}^{\prime}, k_{A}, m_{A}}^{d i h}$ can be derived for the $(-1)^{J+P}=1$ case. Note that the $K \equiv m_{A}$ constraint is applied everywhere. $I^{\text {dih }}$ (the subscripts and variables $\left(\beta_{A}, \theta_{A}\right)$ are dropped for brevity) is the integral over the dihedral angles. Since the potential does not depend on $\alpha_{A}$, integrating over $\alpha_{A}$, gives zero unless $m_{A}=m_{A}^{\prime}$ ( and $\left.K=K^{\prime}\right) . I^{d i h}$ is similar to the corresponding integral for methane.[62] There are three cases:

(a) $K=0$ and $(-1)^{J+P}=1$ case,

$$
I^{d i h}=\left(\frac{1}{\pi}\right) N_{k_{A}^{\prime}, 0} N_{k_{A}, 0} \int_{0}^{2 \pi} d \gamma_{A} \cos \left(k_{A}^{\prime} \gamma_{A}\right) \cos \left(k_{A} \gamma_{A}\right) V\left(\theta_{A}, \beta_{A}, \gamma_{A}\right)
$$

(b) $K=0$ and $(-1)^{J+P}=-1$ case,

$$
I^{d i h}=\left(\frac{1}{\pi}\right) \int_{0}^{2 \pi} d \gamma_{A} \sin \left(k_{A}^{\prime} \gamma_{A}\right) \sin \left(k_{A} \gamma_{A}\right) V\left(\theta_{A}, \beta_{A}, \gamma_{A}\right)
$$

(c) $K>0$ and $(-1)^{J+P}=1$ or -1 case,

$$
I^{d i h}=\left(\frac{1}{2 \pi}\right) \int_{0}^{2 \pi} d \gamma_{A} \cos \left[\left(k_{A}^{\prime}-k_{A}\right) \gamma_{A}\right] V\left(\theta_{A}, \beta_{A}, \gamma_{A}\right)
$$


Eq. (38) is evaluated with Gauss-Legendre quadrature and Eq.(39) is evaluated with equidistant quadrature points one of which is at zero. Although if $k_{A}+k_{A}^{\prime}$ is odd, $\Theta_{l_{A}^{\prime}}^{k_{A}^{\prime}}\left(\theta_{A}\right) \Theta_{l_{A}}^{k_{A}}\left(\theta_{A}\right)$ is not a polynomial in $\cos \theta_{A}$, the Gauss-Legendre quadrature is sufficiently

accurate when the number of points is large enough. If $k_{A}+k_{A}^{\prime}$, is odd, $\bar{d}_{m_{A} k_{A}^{\prime}}\left(\beta_{A}\right) \bar{d}_{m_{A} k_{A}}^{j_{A}}\left(\beta_{A}\right)$ is also not a polynomial in $\cos \beta_{A}$, but the Gauss-Legendre quadrature is sufficiently accurate when the number of points is large enough. It is important that the overlap integrals computed with the quadrature be exact when the number of points is large enough. This was discussed by Leforestier.[7]

Like the KEO matrix elements, the integrals of this section are not actually calculated, but we use the equations of this section to evaluate the matrix-vector products required to use the Lanczos algorithm to compute the spectrum. Potential matrix-vector products are evaluated by doing sums sequentially. $[34,63]$ The equations we use are similar to those of section II C used for methane. [62] The differences are: 1) methane has three $\theta$-type angles and in this paper there are two, $\theta_{A}$ and $\left.\beta_{A} ; 2\right)$ for $\beta_{A}$, the basis functions in the integrand of Eq. (38) are not associated Legendre functions but $\bar{d}_{m_{A} k_{A}}^{j_{A}}\left(\beta_{A}\right)$ functions; 3) there is no $m_{A}^{\prime}$ in Eq. (39) because $m_{A}^{\prime}=m_{A}$. Fast Fourier transforms are used to accelerate the evaluation of the $I^{\text {dih }}[64]$.

\section{USING MONOMER VIBRATIONAL WAVEFUNCTIONS AS BASIS FUNC- TIONS FOR THE DIMER}

The simplest way of computing the rovibrational spectrum of a VDW molecule with flexible monomers is to use polyspherical coordinates and an angular basis whose functions are products of of an associated Legendre function (for $\theta_{1}$ ), spherical harmonics (for the $\theta_{c}, \phi_{c}$ pairs), and a Wigner function (for rotation). The disadvantage of this approach is that many basis functions are required. Instead, it is better to use the BAST KEO and a basis like that of Eq. (20). This approach is better because coupling between the intramolecular angle $\theta_{A}$ and the intermolecular angles $\alpha_{A}, \beta_{A}$, and $\gamma_{A}$ is not important for many VDW molecules. It is not important, mostly because intramolecular frequencies are often much larger than intermolecular frequencies. In this section we go one step further and take full advantage of the BAST KEO by using it with a monomer vibrational wavefunction (MVW) basis. 
Rather than using basis functions for the monomer that are products of functions of single monomer vibrational coordinate (e.g. $\eta_{1} \eta_{2} \eta_{3}$ in section IV B 2), we use eigenfunctions of a monomer vibrational Hamiltonian. These are excellent basis functions. It is common when studying VDW molecules to "neglect" the flexibility of the monomers and to use (ground state) averaged monomer rotational constants. This is equivalent to assuming that a single MVW function is sufficient. In this section, we outline the ideas for a VDW dimer for which one of the monomers is an atom, however, the same ideas can be used for trimers and for molecules with two or three molecular monomers. When both the monomers are diatomics, it is much easier to use a MVW basis, [65] since the orientation of the MF frame is obvious and one only needs to deal with centrifugal coupling terms. In this paper, because the monomer is a triatomic, there are also Coriolis terms and an angular singularity and it is useful to consider different embeddings of the MF frame.

For a dimer composed of $\mathrm{H}_{2} \mathrm{O}$ and an atom, each of the contracted basis functions is a product of an $\mathrm{H}_{2} \mathrm{O}$ vibrational wavefunction, a DVR function for $r_{0}$, a Wigner function that depends on the inter-monomer Euler angles $\left(\alpha_{A}, \beta_{A}, \gamma_{A}\right)$, and a Wigner function that depends on the (two) Euler angles $(\alpha, \beta)$ that specify the orientation of the DF frame,

$$
|v\rangle\left|j_{A} k_{A}\left(m_{A}\right)\right\rangle|J K\rangle
$$

with $K \equiv m_{A}$. We actually use

$$
|v\rangle u_{j_{A} k_{A}\left(m_{A}\right) ; K}^{J P}
$$

which are PA functions because all MVWs have even parity. The potential only depends on the six coordinates $\left(\boldsymbol{q}_{A}, \beta_{A}, \gamma_{A}, r_{0}\right)$ where $\boldsymbol{q}_{A}$ are the three fast intra-monomer coordinates and $\left(\beta_{A}, \gamma_{A}, r_{0}\right)$ are three slow inter-monomer coordinates. The MVW are themselves computed by solving a monomer vibrational Hamiltonian,

$$
H_{v}^{A}=T_{v}^{A}+V^{A}
$$

where the monomer potential $V^{A}$ is obtained by setting inter-monomer coordinates $\left(\beta_{A}, \gamma_{A}, r_{0}\right)$ to reference values in the potential (it is also possible to use the free monomer potential). In our calculations, the reference values we use are those at the top of the barrier separating the two wells. $V^{A}\left(\boldsymbol{q}_{A}\right)=V\left(\boldsymbol{q}_{A}, \beta_{A}^{\text {ref }}, \gamma_{A}^{\text {ref }}, r_{0}^{\text {ref }}\right)$ and $V\left(\boldsymbol{q}_{A}, \beta_{A}, \gamma_{A}, r_{0}\right)=$ $V^{A}\left(\boldsymbol{q}_{A}\right)+\Delta V\left(\boldsymbol{q}_{A}, \beta_{A}, \gamma_{A}, r_{0}\right)$. We use the top of the barrier because it enables us to exploit 
the symmetry of the corresponding MVWs. One could also use the MVW for free $\mathrm{H}_{2} \mathrm{O}$. This would be a good choice for a VDW system, but it would be a poor choice for $\mathrm{H}_{2} \mathrm{O}_{-} \mathrm{Cl}^{-}$, the example molecule of this this paper, because $\mathrm{H}_{2} \mathrm{O}$ in $\mathrm{H}_{2} \mathrm{O}-\mathrm{Cl}^{-}$is rather distorted (e.g. the ionic $\mathrm{OH}$ stretch frequency is red-shifted by about $500 \mathrm{~cm}^{-1}$ ). The monomer problem is solved in a basis that does not have an index which is shared with the inter-monomer basis functions. We use the DVR basis $|\eta\rangle$ for $\boldsymbol{q}_{A}$ where $\eta=\left(\eta_{1}, \eta_{2}, \eta_{3}\right)$ is a composite DVR index,

To use the basis of Eq. (44), we must know how to compute matrix elements of $H=$ $H_{v}^{A}+\Delta V+T_{i n t}+T_{v r}^{A}$. $H_{v}$ is diagonal in the Eq. (44) basis. Consider next matrix elements of $\Delta V$. They are diagonal in the DVR index for $r_{0}$, but the DVR label is not indicated in what follows. The potential matrix elements are

$$
\left\langle u_{j_{A}^{\prime} k_{A}^{\prime}\left(m_{A}\right) ; K}^{J P}\left|\left\langle v^{\prime}\left|\Delta V\left(\boldsymbol{q}_{A}, \beta_{A}, \gamma_{A}, r_{0}\right)\right| v\right\rangle\right| u_{j_{A} k_{A}\left(m_{A}\right) ; K}^{J P}\right\rangle
$$

Because $|v\rangle$ is computed in a DVR basis $|\eta\rangle$, the monomer-part of the integral of Eq. (46) can be computed by using the DVR basis $|\eta\rangle$

$$
\sum_{\eta}\left\langle v^{\prime} \mid \eta\right\rangle\left\langle\eta ; u_{j_{A}^{\prime} k_{A}^{\prime}\left(m_{A}\right) ; K}^{J P}\left|\Delta V\left(\left(\boldsymbol{q}_{A}\right)_{\eta}, \beta_{A}, \gamma_{A}, r_{0}\right)\right| \eta ; u_{j_{A} k_{A}\left(m_{A}\right) ; K}^{J P}\right\rangle\langle\eta \mid v\rangle
$$

The integral in the middle of Eq. (47) is related to the integral we encountered when using the flexible PA basis in section VI. It is diagonal in $\eta$ and computed by removing the integral over $\theta_{A}$ from Eq. (38). We need to store $\Delta V\left(\boldsymbol{q}_{A}, \beta_{A}, \gamma_{A}, r_{0}\right)$ on a $6 \mathrm{D}$ grid. For larger VDW molecules, storing $\Delta V$ would require a lot of memory and it would be better to compute and store the 3D integral over fast intra-monomer coordinates in the middle of Eq. (46)

$$
\begin{aligned}
F_{v^{\prime}, v}\left(\beta_{A}, \gamma_{A}, r_{0}\right) & =\left\langle v^{\prime}\left|\Delta V\left(\boldsymbol{q}_{A}, \beta_{A}, \gamma_{A}, r_{0}\right)\right| v\right\rangle \\
& =\sum_{\eta}\left\langle v^{\prime} \mid \eta\right\rangle \Delta V\left(\left(\boldsymbol{q}_{A}\right)_{\eta}, \beta_{A}, \gamma_{A}, r_{0}\right)\langle\eta \mid v\rangle
\end{aligned}
$$

To use Eq. (48), one needs to store $F_{v^{\prime}, v}\left(\beta_{A}, \gamma_{A}, r_{0}\right)$, which, when the size of the MVW basis is small, requires significantly less memory than storing $\Delta V$ on a $6 \mathrm{D}$ grid. This will be the case whenever the coupling between the intra-monomer and the inter-monomer coordinates is unimportant. We have done calculations with and without pre-computing the $\mathbf{F}$ matrix. The $\mathbf{F}$ matrix here is similar to the $\mathbf{F}$ matrix used to compute vibrational levels of methane in a basis of products of contracted bend and stretch functions, $|b s\rangle$. [66] The difference is that in methane the diagonal index labels the DVR points of the (fast) stretch coordinates 
whereas here the diagonal indices label the quadrature points of the (slow) inter-monomer coordinates (strictly speaking $r_{0}$ point is a DVR point and not a quadrature point). When $v=v^{\prime}, F_{v^{\prime}, v}\left(\beta_{A}, \gamma_{A}, r_{0}\right)$ is a vibrationally averaged potential (averaged over the fast monomer vibrational wavefunction) for the slow inter-monomer coordinates. A vibrational averaged potential (VAP) of this type, with $v=0$, has been used to compute rovibrational levels of water dimer. [19] Results obtained with a single VAP will be quite accurate if the spacing between the monomer energy levels is large. The approach we describe in this paper accounts for coupling between different MVW and hence uses several VAPs.

Matrix elements of $T_{\text {int }}$ are in the Wigner basis $\left|j_{A} k_{A}\left(m_{A}\right)\right\rangle|J K\rangle$ are the same as those presented in section III. $T_{\text {int }}$ does not depend on $\mathbf{q}_{\mathbf{A}}$ and is therefore diagonal in the $v$ index. To compute matrix elements of $T_{v r}^{A}$, we use ideas related to those of Ref. 60. Matrix elements of $T_{v r}^{A}$ in the $|v\rangle\left|j_{A} k_{A}\left(m_{A}\right)\right\rangle$ basis are diagonal in $J$ and $K$ and the same as those used to compute rovibrational levels of a triatomic molecule in a basis of products of vibrational wavefunctions and Wigner functions for rotation. The only difference is that for an isolated triatomic the rotational labels are $J, K, M$ and here they are $j_{A}, k_{A}, m_{A}$. Several groups have used a $|v\rangle\left|j_{A} k_{A}\left(m_{A}\right)\right\rangle$ basis to compute rovibrational levels of isolated molecules. [60, 67-69] The non-zero matrix elements are (to ease the notation $J, K$ labels are dropped),

$$
\begin{aligned}
& \left\langle v^{\prime} ; j_{A}, k_{A},\left(m_{A}\right)\left|T_{v r}^{A}\right| v ; j_{A}, k_{A},\left(m_{A}\right)\right\rangle \\
= & \left\langle v^{\prime}\left|\frac{1}{4}\left(G_{x x}+G_{y y}\right)\right| v\right\rangle\left[j_{A}\left(j_{A}+1\right)-k_{A}^{2}\right]+\left\langle v^{\prime}\left|\frac{1}{2} G_{z z}\right| v\right\rangle k_{A}^{2} \\
& \left\langle v^{\prime} ; j_{A}, k_{A}+2,\left(m_{A}\right)\left|T_{v r}^{A}\right| v ; j_{A}, k_{A},\left(m_{A}\right)\right\rangle \\
= & \left\langle v^{\prime}\left|\frac{1}{4}\left(G_{x x}-G_{y y}\right)\right| v\right\rangle \lambda_{j_{A}, k_{A}}^{+} \lambda_{j_{A}, k_{A}+1}^{+} \\
& \left\langle v^{\prime} ; j_{A}, k_{A}+1,\left(m_{A}\right)\left|T_{v r}^{A}\right| v ; j_{A}, k_{A},\left(m_{A}\right)\right\rangle \\
= & \left\langle v^{\prime}\left|\frac{1}{2} G_{x z}\right| v\right\rangle\left(k+\frac{1}{2}\right) \lambda_{j_{A}, k_{A}}^{+}+\left\langle v^{\prime}\left|\frac{i}{2}\left(p_{\theta}^{H} G_{y, \theta}+G_{y, \theta} p_{\theta}^{H}\right)\right| v\right\rangle \frac{1}{2} \lambda_{j_{A}, k_{A}}^{+} \lambda_{j_{A}, k_{A}}^{+} .
\end{aligned}
$$

Matrices representing $G_{x x}+G_{y y}, G_{x x}-G_{y y}, G_{z z}, G_{x z}$ and $p_{\theta}^{H} G_{y, \theta}+G_{y, \theta} p_{\theta}^{H}$ in the $|v\rangle$ basis are computed and stored before starting the Lanczos calculation. The last matrix is obtained from a product approximation.

Terms in $T_{v r}^{A}$ couple intra- and inter-monomer coordinates and give rise to off-diagonal matrix elements in the MVW basis. In the bisector-z embedded Radau KEO, the Coriolis term and the centrifugal $j_{x}^{\mathrm{MF}, A} j_{z}^{\mathrm{MF}, A}$ terms are zero at equilibrium (see Eq. (22)). In 
previous calculations of VDW molecules that account for flexibility of the monomers the Coriolis term is dropped and because only one vibrational state was retained (albeit a better vibrational state since an adiabatic approximation is used), coupling between different monomer vibrational states due to the centrifugal terms $G_{\alpha \beta} J_{\alpha} J_{\beta}$ was not accounted for. $[44,45]$ We shall show that for $\mathrm{Cl}^{-}-\mathrm{H}_{2} \mathrm{O}$ the Coriolis terms cause errors of about $3 \mathrm{~cm}^{-1}$. Neglecting off-diagonal matrix elements in the MVW basis of centrifugal terms (with two angular momentum operators) has a larger effect.

We now discuss the symmetry of the MVW basis. Under the (12) operation, it can be shown that

$$
\left.(12)|v\rangle\left|u_{j_{A} k_{A}\left(m_{A}\right) ; K}^{J P}=s(v)(-1)^{k_{A}}\right| v\right\rangle \mid u_{j_{A} k_{A}\left(m_{A}\right) ; K}^{J P}
$$

where $s(v)=1$ if $v$ is symmetric under (12) and $s(v)=-1$ if $v$ is anti-symmetric under (12). Even and odd symmetric states are labelled $A+$ and $A-$ and even and odd anti-symmetric states are labelled $B+$ and $B-$ in the $G_{4}$ PI group. Thus, a $G_{4}$ symmetry-adapted basis for each irrep can be easily constructed without linearly combining basis functions.

\section{RESULTS}

In this section, we test the two new methods proposed in this paper by computing rovibrational levels of $\mathrm{Cl}^{-} \mathrm{H}_{2} \mathrm{O}$. Both methods use the BAST KEO, in which the three inter-monomer coordinates are Euler angles. The first method uses uncontracted basis functions. We test three options: Radau/bisector/SKF, Radau/bisector/DVR, and Radau/Eckart/DVR. The

Radau/bisector/SKF approach is described in section IV B1, the Radau/bisector/DVR approach is described in section IV B 2, and the Radau/Eckart/DVR is described in section IV C. The second method uses the MVW basis described in section VII. The results of Ref. 70, obtained with the well-established polyspherical/spherical harmonic-type (SH) basis are used as a benchmark.

\section{A. Uncontracted basis results}

Converged rovibrational levels with $J \leq 2$ on the Rheinecker and Bowman PES[71, 72] have been computed with the well established polyspherical/SH basis approach.[70]. The 
$J=0$ levels are in Tables IV and V of Ref. 70 and the $J=1$ and 2 levels are in Table VI of Ref. 70. In the polyspherical/SH method, $\mathrm{H}_{2} \mathrm{O}$ is represented with two vectors $\vec{r}_{1}$ and $\vec{r}_{2}$. The SH basis functions have non-zero amplitude in all of angular configuration space, but there is significant coupling between the functions. If the coupling between intra- and inter-monomer coordinates is weak, then the two $\mathrm{H}_{2} \mathrm{O}$ vectors should move in concert. To describe that motion many $\mathrm{SH}$ basis functions are required. On the other hand, it is naturally described in the bases of Eq. (20) and Eq. (29).

For the stretch coordinates, we use the same basis sets as in Ref. 70. $N_{R_{1 A}}=N_{R_{2 A}}=7$, and $N_{r_{0}}=24$ PODVR (potential optimized discrete variable representation)[73, 74] functions are used, obtained from the cut potential at the saddle point. Parameters for all the bases are given in Table II. Note that the Radau/bisector/SKF and Radau/bisector/DVR bases are both used with the BAST KEO. For all the $J=0$ levels of Table III, the same results are obtained with the Radau coordinate bisector KEO in both the SKF and DVR bases. The results agree with those computed with the benchmark polyspherical/SH basis. The Radau/bisector/SKF and Radau/bisector/DVR approaches also give the same $J=1$ and 2 levels up to $500 \mathrm{~cm}^{-1}$ and they agree with the polyspherical/SH levels published in Table VI of Ref. 70. The size of the polyspherical/SH and Radau/SKF bases is equal for $J=0$ and $J>0$. The Radau/bisector/DVR basis is larger than the Radau/bisector/SKF basis because a basis index is shared between the monomer vibration and rotation bases in the Radau/bisector/SKF basis. The polyspherical/SH spectral range is the smallest because it is the only approach with no singular integrals. See $E_{\max }$ of Table III, which shows that the spectral range of the polyspherical/SH approach is 40 times smaller than that of the Radau/bisector/DVR approach. The spectral range is important when an iterative eigensolver is used. It is interesting to compare the size of the quadrature grids of the Radau/bisector/SKF and polyspherical/SH approaches. If no ceiling is imposed, the two quadrature grids have the same number of points. However, to reduce the spectral range we impose a ceiling by replacing all potential values above $V_{\text {ceil }}$ with $V_{\text {ceil }}$. [75] Because Gauss Legendre points are denser near the edges (i.e. near 0 and $\pi$ ) and the potential is large when $\theta_{A}$ is small, the number of quadrature points, $N_{\text {quad }}$, under the ceiling value is smaller in the Radau/bisector/SKF case. Overall, the polyspherical/SH and Radau/bisector/SKF approaches are about equally good. The Radau/bisector/SKF approach would be more 
efficient if the intra-monomer and inter-monomer coupling were weaker.

\section{B. Contracted basis results}

Due mostly to the large spacing of the monomer vibrational levels, coupling between intra- and inter-monomer coordinates is unimportant and a small number of MVW basis functions ought to be sufficient. Using the MVW basis rather than the primitive DVR basis, from which the MVWs are computed, therefore makes it possible to significantly reduce the size of the basis required to compute rovibrational levels of a VDM molecule. The reduction is large for $\mathrm{Cl}^{-} \mathrm{H}_{2} \mathrm{O}$ and will, in general, be larger for dimers composed of two molecular monomers. For many VDW molecules, levels obtained with a single MVW function are

quite accurate. In the contracted approach, the basis size is $N_{\text {nbas }}=N_{v} N_{\text {rigid }}$, where $N_{v}$ is the number of MVWs and $N_{\text {rigid }}$ is the number of the functions for the inter-monomer and rotational coordinates, which is the basis size one would have the monomer were assumed to be rigid (hence the choice of the subscript). $N_{\text {rigid }}=N_{r_{0}} N_{\text {Wigner }}$ where $N_{r_{0}}$ is the number of DVR basis functions for $r_{0}$ and $N_{\text {Wigner }}$ is the size of parity-adapted basis of Eq. (33). In this paper, we use $N_{r_{0}}=24, N_{\text {rigid }}=8424$, and $N_{\text {Wigner }}=351$ for $J=0$.

Levels computed with Radau coordinates, the bisector- $z \mathrm{MF}$, and the contracted basis method are presented in Table III for $J=0$ and in Supplementary Material (SM) Table I and SM Table II for $J=1$. Only $N_{v}=70 \mathrm{MVW}$ are required to converge the $J=0$ vibrational levels to within $0.01 \mathrm{~cm}^{-1}$, whereas $N_{\eta}=N_{R_{1 A}} N_{R_{2 A}} N_{\theta_{A}}=1323$ uncontracted DVR functions (Eq. (29)) are required to achieve the same accuracy (the comparison might be slightly less favourable if the DVR basis were pruned). The contracted basis is much more efficient; its size is a factor of 19 smaller. Note that for $\mathrm{H}_{2} \mathrm{O}_{-} \mathrm{Cl}^{-}$, which is fairly rigid, using only a single MVW produces large errors, even keeping all the MVW up to about $6750 \mathrm{~cm}^{-1}$ (roughly two quanta of the $\mathrm{H}_{2} \mathrm{O}$ stretch vibration) is not enough. We have also calculated the same levels with an Eckart MF frame and the $J=0$ levels are shown in Table IV. The accuracy of the levels computed with the Eckart and bisector-z frames is similar. With the same contracted basis $\left(N_{v}=70\right), J=1$ levels are converged to within $0.003 \mathrm{~cm}^{-1}$ of the benchmark values (SM Table I), achieving even better accuracy.

Finally, we evaluate the importance of matrix elements of $T_{v r}^{A}$ that are off-diagonal in 
the MVW basis. There are two kinds of terms that have off-diagonal elements: Coriolis terms $G_{k \alpha} p_{k} J_{\alpha}$ and centrifugal terms $G_{\alpha \beta} J_{\alpha} J_{\beta}$. If the Coriolis terms are neglected, then with $N_{v}=100$ the zero point energy (ZPE) is shifted up by about $3 \mathrm{~cm}^{-1}$ and the excited state energies relative to the ZPE are shifted down by as much as $2 \mathrm{~cm}^{-1}$, for levels up to $1000 \mathrm{~cm}^{-1}$, as shown in the penultimate Table III. The error introduced by neglecting the off-diagonal centrifugal terms is larger. The ZPE is shifted up by more than $5 \mathrm{~cm}^{-1}$ and the relative excited state energies are shifted by up to $11 \mathrm{~cm}^{-1}$, for levels up to $1000 \mathrm{~cm}^{-1}$, as shown in the last column of Table III.

\section{CONCLUSION}

VDW molecules have been avidly studied for many years. To analyse their spectra and extract information about PESs one must solve a rovibrational Schroedinger equation. Owing to the disparity of the intra- and inter-monomer frequencies, this is frequently done by assuming that the monomers of which the VDW is composed are rigid. This approximation frequently works well. However, to understand how monomer vibrations are affected by the VDW bond one obviously needs to include them in the theoretical treatment. Even if only transitions between inter-monomer levels are important, for an accurate analysis one must include the monomer vibrations. Doing this is difficult because it adds many coordinates and therefore significantly increases the cost of solving the Schroedinger equation.

To solve the rovibrational Schroedinger equation one must choose coordinates and a basis set, and compute eigenvalues and eigenvectors. It is always possible to use polyspherical coordinates, a frame attached to two of the vectors used to define the coordinates, and an angular basis composed of products of spherical harmonics and a Wigner rotational function. $[28,70]$ This is the approach we call $\mathrm{SH}$ in this paper. Its main disadvantage is that many basis functions are required because coupling between the polyspherical coordinates is important. For VDW dimers it is is better to use intra-monomer coordinates to describe the vibration of the monomers, the distance between the two monomers that make up the dimer, and Euler angles that specify the orientation of the monomers with respect to a frame attached to the inter-monomer vector. BAST derived the KEO in these coordinates. [42] In these coordinates coupling is less important because they exploit the natural separation 
between slow inter-monomer and fast intra-monomer motion.

We have used the BAST KEO to compute rovibrational energy levels of $\mathrm{H}_{2} \mathrm{O}-\mathrm{Cl}^{-}$. This is the first time that the BAST KEO has been used, without making approximations, to compute rovibrational energy levels of a dimer. To deal with the singularity in $T_{\text {int }}$, the term in the KEO that couples intra-monomer and inter-monomer coordinates, we use a basis each of whose functions includes a product of a Wigner function for $\mathrm{H}_{2} \mathrm{O}$ and a Wigner function for the overall rotation of the molecule and where $m_{A}=K$. This is standard practice also for rigid-monomer calculations. To deal with singularities in the monomer $\mathrm{KEO}, T^{A}$, we use a basis with another shared quantum number, $k_{A}$. Using the BAST KEO, one can take advantage of the weakness of the coupling between inter-monomer and intra-monomer coordinates by taking a maximum value of $j_{A}$ smaller than the maximum value of $l_{A}$ (where $l_{A}$ labels a vibrational Legendre basis function (Eq. (20)). Because the monomer singularities are not important for the levels we compute it is also possible to use a basis for the vibration of the $\mathrm{H}_{2} \mathrm{O}$ monomer that does not have a shared index, e.g. a DVR monomer basis. When a DVR is used, it is possible to discard DVR functions centered at high-lying points.

To fully exploit the separation of the inter- and intra-monomer coordinates in the BAST KEO, we have used contracted monomer vibrational wavefunctions as basis functions. This enables us to reduce the size of the basis by about a factor of 20. For dimers with weaker inter-intra coupling, two molecular monomers, or for which one wishes states with greater monomer excitation, the basis reduction will be larger. Some of the ideas of this paper are similar to those used for water dimer by Leforestier et al. [44, 45], however, there are important differences. Leforestier et al. use an adiabatic approximation whereas we use simplified contracted functions (or a "crude adiabatic" approximation). [35, 76] The adiabatic basis is better, but its calculation is costly and it would be extremely difficult to include coupling between the adiabatic vibrational states. Leforestier et al. neglect not only non-adiabatic coupling but also centrifugal and Coriolis terms. We retain all coupling between the monomer vibrational states. For water dimer the method of Leforestier et al. works well, but for other dimers some of the approximations will be less accurate. For $\mathrm{H}_{2} \mathrm{O}$ $\mathrm{Cl}^{-}$, neglecting Coriolis and centrifugal coupling shifts energy levels by about $5 \mathrm{~cm}^{-1}$. We obviate the need to store the potential on a full-d grid by using the F matrix idea. [76] For $\mathrm{H}_{2} \mathrm{O}-\mathrm{Cl}^{-}$this is not necessary, but for larger VDW molecules it will make contracted- 
basis calculations practical. By increasing the number of Lanczos iterations, it would be possible to compute monomer frequency shifts. In this paper we demonstrate that the ideas by work by computing lower energy levels. Using these ideas it will be possible to compute rovibrational spectra of larger VDW molecules without approximations.

\section{SUPPLEMENTARY MATERIAL}

In the Supplementary Material we give errors of $J=1$ levels with $N_{v}=70$ MVW basis functions.

\section{Acknowledgments}

This work was supported by the Natural Sciences and Engineering Research Council of Canada. Calculations were done on computers of the Compute Canada network. 


\section{APPENDIX A: SOME ONE-DIMENSIONAL BEND INTEGRALS REQUIRED FOR THE SKF MONOMER BASIS CALCULATIONS}

One dimensional bend integrals are defined by:

$$
\begin{aligned}
A_{l^{\prime}, l, m} & =\left\langle\Theta_{l^{\prime}}^{m}\left|\frac{1}{\sin ^{2} \theta}\right| \Theta_{l}^{m}\right\rangle \\
B_{l^{\prime}, l, m} & =\left\langle\Theta_{l^{\prime}}^{m}|\cot \theta| \Theta_{l}^{m}\right\rangle \\
C_{l^{\prime}, l, m} & =\left\langle\Theta_{l^{\prime}}^{m+1} \mid \Theta_{l}^{m}\right\rangle \\
D_{l^{\prime}, l, m} & =\left\langle\Theta_{l^{\prime}}^{m+1}|\cot \theta| \Theta_{l}^{m}\right\rangle . \\
E_{l^{\prime}, l, m} & =\left\langle\Theta_{l^{\prime}}^{m}\left|\frac{1}{1+\cos \theta}\right| \Theta_{l}^{m}\right\rangle \\
F_{l^{\prime}, l, m} & =\left\langle\Theta_{l^{\prime}}^{m+2}\left|\frac{1}{1+\cos \theta}\right| \Theta_{l}^{m}\right\rangle \\
G_{l^{\prime}, l, m} & =\left\langle\Theta_{l^{\prime}}^{m+1}\left|\frac{1}{\sin \theta}\right| \Theta_{l}^{m}\right\rangle \\
H_{l^{\prime}, l, m} & =\left\langle\Theta_{l^{\prime}}^{m+2} \mid \Theta_{l}^{m}\right\rangle \\
I_{l^{\prime}, l, m} & =\left\langle\Theta_{l^{\prime}}^{m}\left|\frac{1}{1-\cos \theta}\right| \Theta_{l}^{m}\right\rangle \\
J_{l^{\prime}, l, m} & =\left\langle\Theta_{l^{\prime}}^{m+2}\left|\frac{1}{1-\cos \theta}\right| \Theta_{l}^{m}\right\rangle .
\end{aligned}
$$

A, B and $\mathbf{C}$ were previously used for triatomic [57] and larger molecules[77] when a $\Theta_{l_{A}}^{0}\left(\theta_{A}\right)$ basis was used for $\theta_{A}$ (no index is shared). We use two types of Gauss quadratures to evaluate these integrals. Although we use quadrature, they are all exact except for those that are singular (A, E, I). Integrals of B, C, are computed exactly with a Gauss Chebyshev quadrature with $l_{x}+1$ points; $l_{x}$ is the maximum value of $l$. All other integrals are computed with a Gauss Legendre quadrature with $l_{x}+1$ points (some integrals could also be evaluated exactly with fewer points). However, the integrands of $\mathbf{A}$ (at $\theta=0$ and $\pi$ ), $\mathbf{E}$ (at $\theta=\pi$ ) and $\mathbf{I}($ at $\theta=0)$ are singular for $m=0$. They are nevertheless evaluated with $n_{\text {sing }}=l_{x}+1$ Gauss Legendre quadrature points. We have shown that using more Gauss Legendre points can give more accurate levels when wavefunctions have amplitude near the singular point[57]. But when wavefunctions do not have amplitude near the singular point(s), which is the case in this paper, $n_{\text {sing }}=l_{x}+1$ is a good choice. 


\section{APPENDIX B: THE EFFECT OF $E^{*}$ ON THE INTER-MONOMER AND INTRA- MONOMER COORDINATES}

To make parity-adapted basis functions, we need to determine the effect of the inversion operation $E^{*}$ on the inter-monomer coordinates (Euler angles) and intra-monomer coordinates. There are three ways to define how these coordinates behave under $E^{*}$. In this appendix we present the three possible cases and and explain why we prefer Case II.

Denote the coordinates of a vector $\mathbf{z}_{i}$ of monomer $A\left(x_{i}, y_{i}, z_{i}\right)^{t}$ in the MF frame and $\left(X_{i}, Y_{i}, Z_{i}\right)^{t}$ in the SF frame (to ease the notation we drop the $A$ label on the components). The MF frame for monomer $A$ is obtained by rotating the SF frame by two Euler angles $(\alpha, \beta)$, and then rotating the resulting frame (DF frame) by three Euler angles $\left(\alpha_{A}, \beta_{A}, \gamma_{A}\right)$. We find the relation,

$$
\left(\begin{array}{c}
x_{i} \\
y_{i} \\
z_{i}
\end{array}\right)=R_{z}\left(\gamma_{A}\right) R_{y}\left(\beta_{A}\right) R_{z}\left(\alpha_{A}\right) R_{y}(\beta) R_{z}(\alpha)\left(\begin{array}{c}
X_{i} \\
Y_{i} \\
Z_{i}
\end{array}\right)
$$

with[32]

$$
R_{z}(\alpha)=\left(\begin{array}{lll}
\cos \alpha & \sin \alpha & 0 \\
-\sin \alpha & \cos \alpha & 0 \\
0 & 0 & 1
\end{array}\right), \quad R_{y}(\beta)=\left(\begin{array}{lll}
\cos \beta & 0 & -\sin \beta \\
0 & 1 & 0 \\
\sin \beta & 0 & \cos \beta
\end{array}\right)
$$

Thus, the SF Cartesian coordinates of a vector of monomer $A$ are completely determined by $\left(x_{i}, y_{i}, z_{i}\right)$ and the five angles $\left(\alpha, \beta, \alpha_{A}, \beta_{A}, \gamma_{A}\right)$.

We shall now determine how $E^{*}$ affects $\left(x_{i}, y_{i}, z_{i}\right)$ and $\left(\alpha, \beta, \alpha_{A}, \beta_{A}, \gamma_{A}\right)$. This is done by using

$$
E^{*}:\left(X_{i}, Y_{i}, Z_{i}\right) \longrightarrow\left(-X_{i},-Y_{i},-Z_{i}\right)
$$

Since the inversion operation always flips $\mathbf{r}_{0}$ whose spherical polar angles in the SF frame are $(\alpha, \beta)$, we find,

$$
E^{*}:(\alpha, \beta) \longrightarrow(\pi+\alpha, \pi-\beta) \text {. }
$$

There are three ways of choosing how the remaining coordinates $\left(x_{i}, y_{i}, z_{i}\right)$ and $\left(\alpha_{A}, \beta_{A}, \gamma_{A}\right)$ are affected by $E^{*}$ that are consistent with Eq. (B1). Each way corresponds to one way of 
choosing how the MF frame axes react to $E^{*}$ :

$$
\begin{array}{r}
\text { Case I, } \quad E^{*}:\left(x_{i}, y_{i}, z_{i}\right) \longrightarrow\left(-x_{i}, y_{i}, z_{i}\right) \\
\text { Case II, } \quad E^{*}:\left(x_{i}, y_{i}, z_{i}\right) \longrightarrow\left(x_{i},-y_{i}, z_{i}\right) \\
\text { Case III, } \quad E^{*}:\left(x_{i}, y_{i}, z_{i}\right) \longrightarrow\left(x_{i}, y_{i},-z_{i}\right) .
\end{array}
$$

The effect on $\left(\alpha_{A}, \beta_{A}, \gamma_{A}\right)$ for each of the three cases is given in Table V. Case II has the useful advantage that with this choice the monomer vibrational coordinates change under $E^{*}$ exactly as they do in the usual polyspherical case where the $z$ axis is along one of the vectors and $y$ is perpendicular to the plane containing two vectors. This means that the vibrational basis used for the free monomer can be used for the dimer because $E^{*}$ does not change $r$ and $\theta$ coordinates and changes the sign of $\phi$ coordinates. This is also the choice made by Leforestier[13] and van der Avoird[14, 15] in their studies of water dimer.

In all three cases, PA functions are proportional to $|\xi\rangle+(-1)^{P} E^{*}|\xi\rangle$, where $E^{*}|\xi\rangle$ is given in the last line of Table V. In Case II, the PA functions are

$$
|\xi\rangle+(-1)^{\left(J+P+k_{A}+k_{B}\right)}|\bar{\xi}\rangle,
$$

where $|\xi\rangle$ and $\bar{\xi}\rangle$ are defined in footnotes of Table V. One can show that the functions of Eq. (B6) are real if $(-1)^{(J+P)}=1$ and imaginary if $(-1)^{(J+P)}=-1$. For a particular $J$, this guarantees that all the PA functions for a given parity are either real or imaginary. Hence the Hamiltonian matrix is guaranteed to be real. In Case I, the PA functions are

$$
|\xi\rangle+(-1)^{(J+P)}|\bar{\xi}\rangle .
$$

For a particular $J$, the PA functions for a given parity are real if $k_{A}+k_{B}$ is even and imaginary if $k_{A}+k_{B}$ is odd. If there are non-zero matrix elements in the PA basis between functions with the same parity one of which is real and other of which is imaginary, then there will be Hamiltonian matrix elements that are not real.

Althorpe and Clary[16] and Chen, Liu, and Light[17] assume rigid monomers and use Case I for $\mathrm{H}_{2} \mathrm{O}$ dimer. Owing to the fact that there are no matrix elements between real and imaginary PA functions all of their matrix elements are real. This is only true because PA functions that are real are symmetric with respect to permutation of the two $\mathrm{H}$ atoms of an $\mathrm{H}_{2} \mathrm{O}$ monomer $\left(k_{A}+k_{B} j\right.$ even) and $\mathrm{PA}$ functions that are imaginary are antisymmetric with 
respect to permutation of the two $\mathrm{H}$ atoms $\left(k_{A}+k_{B}\right.$ odd). For a non-symmetric monomer (e.g. HOD) matrix elements between real and imaginary PA functions would be non-zero. Case III was used by Van der Avoid and co-workers for dimers in which one monomer is $\mathrm{NH}_{3}$. $[3,9,12]$ In this case, an advantage of Case III is that the z-axis of the MF frame is flipped by $E^{*}$. There is then a single vibrational coordinate that changes sign when $\mathrm{NH}_{3}$ is inverted. However, the same advantage can be obtained with a Case II basis because only the dihedral polyspherical angle changes sign. Note also that the signs of both the $k_{F}$ and $m_{F}$ labels of the ket in the last line of Table $\mathrm{V}$ are changed in Case I and II, but that only the sign of the $m_{F}$ label is changed in Case III. Finally the transformation property of $\left(\alpha_{B}, \beta_{B}, \gamma_{B}\right)$ under inversion operation is the same as that of $\left(\alpha_{A}, \beta_{A}, \gamma_{A}\right)$ and not included in Table $\mathrm{V}$.

[1] B L Blaine, and G E Ewing Annual Review of Physical Chemistry 27, 553 (1976)

[2] D. J. Seabird, Chem. Rev. 88, 843 (1988).

[3] A. van der Avoird, P. E. S. Wormer, and R. Moszynski, Chem. Rev. 94, 1931 (1994).

[4] P.E.S. Wormer and A. van Dev Avoird, Chem. Rev. 100, 4109 (2000).

[5] G. Brocks and T. Huygen, J. Chem. Phys. 85, 3411 (1986)

[6] J. Hutson, J. Chem. Phys. 92, 157 (1990)

[7] C. Leforestier, J. Chem. Phys. 101, 7357 (1994).

[8] J. W. I. van Bladel, A. van der Avoird, and P. E. S. Wormer, J. Chem. Phys. 94, 501 (1991)

[9] J. W. I. van Bladel, A. van der Avoird, and P. E. S. Wormer, J. Phys. Chem. 95, 5414 (1991).

[10] T. G. A. Heijmen, P. E. S. Wormer, A. van der Avoird, R. E. Miller, and R. Moszynski, J. Chem. Phys. 110, 5639 (1999)

[11] E. H. T. Olthof, A. van der Avoird, and P. E. S. Wormer, J. Chem. Phys. 101, 8430 (1994)

[12] E. H. T. Olthof, A. van der Avoird, and P. E. S. Wormer, J. G. Loeser, and R. J. Saykally J. Chem. Phys. 101, 8443 (1994)

[13] C. Leforestier, L. B. Braly, K. Liu, M. J. Elroy, and R. J. Saykally, J. Chem. Phys. 106, 8527 (1997)

[14] G. C. Groenenboom, P. E. S. Wormer, A. van der Avoird, E. M. Mas, R. Bukowski, and K. 
Szalewicz, J. Chem. Phys. 113, 6702 (2000)

[15] M. J. Smit, G. C. Groenenboom, P. E. S. Wormer, and A. van der Avoird, R. Bukowski, and K. Szalewicz, J. Phys. Chem. 105, 6212 (2001).

[16] S. C. Althorpe and D. C. Clary, J. Chem. Phys. 101, 3603 (1994).

[17] H. Chen, S. Liu, and J. C. Light, J. Chem. Phys. 110, 168 (1999).

[18] Y.-B. Duan, R. Wang, and I. Mukhopadhyay, Chem. Phys. 280, 119 (2002).

[19] R. E. A. Kelly, J. Tennyson, G. C. Groenenboom, and A. van der Avoird, J. Quant. Spectrosc. Radiat. Transfer. 111, 1262 (2010).

[20] J. Tennyson, M. J. Barber, and R. E. A. Kelly, Phil. Trans. R. Soc. A 370, 2656 (2012).

[21] Ad van der Avoird, R. Podeszwa, K. Szalewicz, C. Leforestier, R. van Harrevelt, P. R. Bunker, M. Schnell, G. von Helden and G. Meijer, Phys. Chem. Chem. Phys. 12, 8219 (2010)

[22] X.-G. Wang and T. Carrington, Jr., J. Chem. Phys. 113, 044313 (2000).

[23] A. van der Avoird and D. J. Nesbitt, J. Chem. Phys. 134, 044314 (2011).

[24] X.-G. Wang, T. Carrington, Jr., J. Tang, and A. R. W. McKellar, J. Chem. Phys. 123, 034301 (2005)

[25] H. Li, P.-N. Roy and R. Le Roy. , J. Chem. Phys. 132, 214309 (2010)

[26] R. Dawes, X.-G. Wang, A. W. Jasper, and T. Carrington, Jr., J. Chem. Phys 133, 144306 (2010)

[27] X.-G. Wang, T. Carrington, Jr., R. Dawes, and A. W. Jasper, J. Mol. Spectrosc. 268, 53 (2011)

[28] X. Chapuisat and C. Iung, Phys. Rev. A 45, 6217 (1992).

[29] F. Gatti and C. Iung, Physics Reports 4841 (2009).

[30] X.-G. Wang and T. Carrington, Jr, J. Chem. Phys. 121, 2937 (2004).

[31] J. C. Light and T. Carrington Jr., Adv. Chem. Phys. 114, 263 (2000).

[32] R. N. Zare, Angular Momentum (Wiley: New York 1988).

[33] C. Iung and C. Leforestier, J. Chem. Phys. 90, 3198 (1989).

[34] M. N. Bramley and T. Carrington, Jr, J. Chem. Phys. 99, 8519 (1993).

[35] M.J. Bramley and T. Carrington J. Chem. Phys. 101, 8494 (1994)

[36] R. B. Lehoucq, S. K. Gray, D.-H. Zhang, and J. C. Light, Comp. Phys. Commn. 10915 (1998).

[37] V. A. Mandelshtam and H. S. Taylor, J. Chem. Phys. 1027390 (1995). 
[38] H.-G. Yu and S. C. Smith, Ber. Bunsenges. Phys. Chem. 101400 (1997).

[39] R. Chen and H. Guo, J. Chem. Phys. 108, 6068 (1998).

[40] G. Avila and T. Carrington, Journal of Chemical Physics 134 054126-1 - 054126-16 (2011)

[41] J. Brown, X.-G. Wang, R. Dawes, and T. Carrington Journal of Chemical Physics $136134306-$ 1 - 134306-12 (2012)

[42] G. Brocks, A. Van Der Avoird, B. T. Sutcliffe, and J. Tennyson, Mol. Phys. 50, 1025 (1983).

[43] F. Gatti, C. Iung, C. Leforestier and X. Chapuisat, J. Chem. Phys. 111, 7236 (1999)

[44] C. Leforestier, F. Gatti, R. S. Fellers, and R. J. Saykally, J. Chem. Phys. 117, 8710 (2002).

[45] C. Leforestier, K. Szalewicz, and A van der Avoird, J. Chem. Phys. 137, 014305 (2012).

[46] F. Gatti, J. Chem. Phys. 111, 7225 (1999)

[47] X.-G. Wang and T. Carrington, Jr., J. Chem. Phys. 113, 7097 (2000).

[48] Zhiqiang Zhao, Jun Chen, Zhaojun Zhang, Dong H. Zhang, David Lauvergnat and Fabien Gatti J. Chem. Phys. 2016, 144, 204302

[49] T. Carrington and X.-G. Wang, Wiley Interdisciplinary Reviews: Computational Molecular Science 1, 952 (2011).

[50] J. Tennyson, Comp. Phys. Rep. 4, 1 (1986).

[51] F.T. Smith, Phys. Rev. Lett. 45, 1157 (1980)

[52] S. Carter, N. C. Handy, and B. T. Sutcliffe, Mol. Phys. 49, 745 (1983).

[53] H. Wei and T. Carrington J. Chem. Phys. 101, 1343 (1994).

[54] P. Sarkar, N. Poulin and T. Carrington, Jr., J. Chem. Phys 110, 10269 (1999)

[55] B. T. Sutcliffe and J. Tennyson Int. J. Quant. Chem. 39, 183 (1991).

[56] B. T. Sutcliffe and J. Tennyson Int. J. Quant. Chem. 42, 941 (1992).

[57] X.-G. Wang and T. Carrington, Mol. Phys. 110, 825 (2012).

[58] H. Wei and T. Carrington, Jr, Chem. Phys. Lett. 287, 289 (1998).

[59] H. Wei and T. Carrington, Journal of Chemical Physics 107 2813-2818 (1997)

[60] X.-G. Wang and T. Carrington, Jr., J. Chem. Phys. 138, 104106 (2013).

[61] P. R. Bunker and P. Jensen, Molecular Symmetry and Spectroscopy (NRC Research Press: Ottawa 1998).

[62] X.-G. Wang and T. Carrington, Jr, J. Chem. Phys. 118, 6946 (2003).

[63] U. Manthe and H. Koeppel, J. Chem. Phys. 93, 345 (1990). 
[64] X.-G. Wang and T. Carrington, Jr, J. Chem. Phys. 123, 154303 (2005).

[65] Dong H. Zhang, Qian Wu, John Z. H. Zhang, Michael von Dirke, and Zlatko Bacic J. Chem. Phys. 102, 2315 (1995).

[66] X.-G. Wang and T. Carrington, Jr, J. Chem. Phys. 119, 101 (2003).

[67] C. Fábri, E. Mátyus, T. Furtenbacher, L. Nemes, B. Mihály, T. Zoltáni, and A. G. Császár, J. Chem. Phys. 135, 094307(2011).

[68] M. Rey, A. V. Nikitin, and V. G. Tyuterev, J. Chem. Phys. 136, 244106 (2012).

[69] R. Wodraszka and U. Manthe J. Phys. Chem. A 117 7246-7255 (2013)

[70] X.-G. Wang and T. Carrington, Jr., J. Chem. Phys. 140, 204306 (2014).

[71] J. Rheinecker and J. M. Bowman, J. Chem. Phys. 124131102 (2006)

[72] J. Rheinecker and J. M. Bowman, J. Chem. Phys. 125133206 (2006)

[73] H. Wei and T. Carrington, Jr., J. Chem. Phys. 97, 3029 (1992).

[74] J. Echave and D. C. Clary, Chem. Phys. Lett. 190, 225 (1992).

[75] M.J. Bramley, J.W. Tromp, T. Carrington and G.C. Corey, J. Chem. Phys. 100, 6175-6194 (1994)

[76] X.-G. Wang and T. Carrington J. Chem. Phys. 117, 6923 (2002)

[77] X.-G. Wang and T. Carrington, Mol. Phys. 111, 2320 (2013). 
TABLE I: The effect of symmetry operations on the coordinates and basis functions for $\mathrm{Cl}^{-} \mathrm{H}_{2} \mathrm{O}$. The stretch part of the basis functions is omitted for simplicity. The effect of $E^{*}$ on the flexible monomer basis functions is independent of the monomer vibrational coordinates.

\begin{tabular}{|c|c|c|c|}
\hline & Coordinate & Rigid monomer Basis & Flexible monomer SKF basis \\
\hline \multicolumn{4}{|c|}{ Radau coordinates } \\
\hline$E$ & $\left(\alpha, \beta ; \alpha_{A}, \beta_{A}, \gamma_{A} ; R_{1 A}, R_{2 A}, \theta_{A}\right)$ & $\left|j_{A}, k_{A},\left(m_{A}\right) ; J K\right\rangle$ & $\left|l_{A} ; j_{A}, k_{A},\left(m_{A}\right) ; J K\right\rangle$ \\
\hline$E^{*}$ & $\left(\pi+\alpha, \pi-\beta ; \pi-\alpha_{A}, \beta_{A},-\gamma_{A} ; R_{1 A}, R_{2 A}, \theta_{A}\right)$ & $(-1)^{J+k_{A}}\left|j_{A}, \bar{k}_{A},\left(\bar{m}_{A}\right) ; J \bar{K}\right\rangle$ & $(-1)^{J}\left|l_{A} ; j_{A}, \bar{k}_{A},\left(\bar{m}_{A}\right) ; J \bar{K}\right\rangle$ \\
\hline$(12)$ & $\left(\alpha, \beta ; \alpha_{A}, \beta_{A}, \gamma_{A}+\pi ; R_{2 A}, R_{1 A}, \theta_{A}\right)$ & $(-1)^{k_{A}}\left|j_{A}, k_{A},\left(m_{A}\right) ; J K\right\rangle$ & $(-1)^{k_{A}}\left|l_{A} ; j_{A}, k_{A},\left(m_{A}\right) ; J K\right\rangle$ \\
\hline \multicolumn{4}{|c|}{ Jacobi coordinates } \\
\hline$E$ & $\left(\alpha, \beta ; \alpha_{A}, \beta_{A}, \gamma_{A} ; R_{A}, r_{A}, \theta_{A}\right)$ & $\left|j_{A}, k_{A},\left(m_{A}\right) ; J K\right\rangle$ & $\left|l_{A} ; j_{A}, k_{A},\left(m_{A}\right) ; J K\right\rangle$ \\
\hline$E^{*}$ & $\left(\pi+\alpha, \pi-\beta ; \pi-\alpha_{A}, \beta_{A},-\gamma_{A} ; R_{1 A}, R_{2 A}, \theta_{A}\right)$ & $(-1)^{J+k_{A}}\left|j_{A}, \bar{k}_{A},\left(\bar{m}_{A}\right) ; J \bar{K}\right\rangle$ & $(-1)^{J}\left|l_{A} ; j_{A}, \bar{k}_{A},\left(\bar{m}_{A}\right) ; J \bar{K}\right\rangle$ \\
\hline$(12)$ & $\left(\alpha, \beta ; \alpha_{A}, \beta_{A}, \gamma_{A}+\pi ; R_{A}, r_{A}, \pi-\theta_{A}\right)$ & $(-1)^{k_{A}}\left|j_{A}, k_{A},\left(m_{A}\right) ; J K\right\rangle$ & $(-1)^{l_{A}}\left|l_{A} ; j_{A}, k_{A},\left(m_{A}\right) ; J K\right\rangle$ \\
\hline
\end{tabular}

TABLE II: Parameters for the uncontracted SH, SKF, and DVR bases. $N_{R_{1 A}}=N_{R_{2 A}}=7$, and $N_{r_{0}}=24$ for all three bases. $1 \mathrm{M}=10^{6}$ and $1 \mathrm{~K}=10^{3}$.

\begin{tabular}{lccc}
\hline \hline & $\mathrm{SH}^{a}$ & SKF & DVR \\
\hline \hline Basis & $\max \left(l_{1}, l_{2}, m_{2}\right)=25$ & $\max \left(l_{A}, j_{A}, k_{A}\right)=25$ & $\max \left(j_{A}, k_{A}\right)=25, N_{\theta_{A}}=27$ \\
Quadrature points & $N_{\theta}=27, N_{\phi}=54$ & $N_{\beta}=27, N_{\gamma}=54$ & $N_{\beta}=27, N_{\gamma}=54$ \\
$N_{\text {bas }}(\mathrm{M})$ & 7.29 & 7.29 & 11.14 \\
$N_{\text {quad }}(\mathrm{M})^{b}$ & 13.32 & 10.20 & 10.20 \\
$E_{\text {max }}(\mathrm{K})^{c}$ & 85 & 2600 & 4320 \\
Angular basis & $\left|l_{1},\left(m_{1}\right), l_{2}, m_{2}\right\rangle|J K\rangle$ & $\left|l_{A}\left(k_{A}\right) ; j_{A}, k_{A},\left(m_{A}\right)\right\rangle|J K\rangle$ & $\left|\eta_{3} ; j_{A}, k_{A},\left(m_{A}\right)\right\rangle|J K\rangle$ \\
& $\left(K \equiv m_{1}+m_{2}\right)$ & $\left(K \equiv m_{A}\right)$ & $\left(K \equiv m_{A}\right)$ \\
\hline \hline
\end{tabular}

${ }^{a}$ SH (spherical harmonic) basis parameters are from Wang and Carrington, JCP, 140, 204306(2014)

${ }^{b} N_{\text {quad }}$ is the number of quadrature/DVR points below a ceiling value of $30000 \mathrm{~cm}^{-1} .{ }^{c} E_{\max }$ is the spectral range of the $A+$ symmetry block. 
TABLE III: $A+$ vibrational levels of $\mathrm{Cl}^{-} \mathrm{H}_{2} \mathrm{O}$ calculated with different methods. All levels are presented as differences relative to the benchmark levels of the second column. The monomerfixed frame attached to $\mathrm{H}_{2} \mathrm{O}$ is the bisector- $z$ frame. The second to last column is obtained by dropping the Coriolis term of $\mathrm{H}_{2} \mathrm{O}$. The last column is obtained by neglecting matrix elements of the centrifugal terms $G_{\alpha \beta} J_{\alpha} J_{\beta}$ in $T_{v r}^{A}$ that are off diagonal in the $v$ index.

\begin{tabular}{|c|c|c|c|c|c|c|c|c|c|}
\hline \multirow[t]{3}{*}{ state $^{a}$} & \multirow[t]{3}{*}{ Ref. $70^{b}$} & \multirow[t]{3}{*}{$\mathrm{SKF}^{c}$} & \multirow{3}{*}{$\begin{array}{l}\mathrm{DVR}^{d} \\
N_{\eta}=1323\end{array}$} & \multicolumn{5}{|c|}{$|v\rangle$ basis $^{e}$} & \multirow{3}{*}{$\begin{array}{r}N_{v}=100 \\
17690 .\end{array}$} \\
\hline & & & & $N_{v}=10$ & $N_{v}=30$ & $N_{v}=70$ & $N_{v}=100$ & $N_{v}=100$ & \\
\hline & & & & $6750 .^{f}$ & 10600. & 15300 & 17690. & 17690. & \\
\hline GS & 5023.765 & 0.000 & 0.000 & 8.444 & 0.186 & 0.003 & 0.000 & 3.081 & 5.483 \\
\hline$v_{1}$ & 196.807 & 0.000 & 0.000 & -0.522 & -0.007 & 0.000 & 0.000 & -0.137 & -0.661 \\
\hline$v_{2}$ & 332.103 & 0.000 & 0.000 & -0.737 & 0.079 & 0.002 & 0.000 & -1.519 & 2.865 \\
\hline $2 v_{1}$ & 387.813 & 0.000 & 0.000 & -0.967 & -0.010 & 0.000 & 0.000 & -0.273 & -1.283 \\
\hline$v_{1}+v_{2}$ & 519.606 & 0.000 & 0.000 & -1.275 & 0.069 & 0.003 & 0.000 & -1.583 & 1.832 \\
\hline $2 v_{2}$ & 571.955 & 0.000 & 0.000 & -1.783 & 0.058 & 0.002 & 0.000 & -2.133 & -0.855 \\
\hline $3 v_{1}$ & 573.258 & 0.000 & 0.000 & -1.477 & -0.004 & 0.001 & 0.000 & -0.554 & 2.333 \\
\hline $2 v_{1}+v_{2}$ & 700.583 & 0.000 & 0.000 & -1.735 & 0.060 & 0.003 & 0.000 & -1.644 & 0.831 \\
\hline $4 v_{1}$ & 751.907 & 0.000 & 0.000 & -1.666 & 0.016 & 0.002 & 0.000 & -1.178 & -2.049 \\
\hline$v_{1}+2 v_{2}$ & 754.384 & 0.000 & 0.000 & -2.296 & 0.027 & 0.002 & 0.000 & -1.607 & 2.352 \\
\hline $3 v_{2}$ & 854.914 & 0.000 & 0.000 & 3.072 & 0.313 & 0.010 & 0.001 & -2.169 & 11.018 \\
\hline $3 v_{1}+v_{2}$ & 875.143 & 0.000 & 0.000 & -2.125 & 0.052 & 0.003 & 0.000 & -1.700 & -0.105 \\
\hline $5 v_{1}$ & 925.563 & 0.000 & 0.000 & -1.872 & 0.009 & 0.002 & 0.000 & -0.846 & -2.728 \\
\hline $2 v_{1}+2 v_{2}$ & 931.216 & 0.000 & 0.000 & -2.685 & 0.028 & 0.002 & 0.000 & -2.034 & 1.947 \\
\hline$N_{\text {bas }}(\mathrm{M})$ & 7.29 & 7.29 & 11.14 & 0.084 & 0.253 & 0.590 & 0.842 & 0.842 & 0.842 \\
\hline$N_{\text {quad }}(\mathrm{M})$ & 13.32 & 10.20 & 10.20 & & & & & & \\
\hline$E_{\max }(\mathrm{K})$ & 85 & 2600 & 4320 & 55 & 67 & 210 & 837 & & \\
\hline
\end{tabular}

a See Ref. 70 for assignment.

${ }^{b}$ Benchmark levels reported in Ref. 70 were computed with polyspherical angles and a spherical harmonic type basis. The Basis I of Ref. 70 is used.

${ }^{c}$ SKF basis of this paper

${ }^{d} N_{\text {bas }}=8424 * N_{\eta}$ where $N_{\eta}=N_{R_{1 A}} N_{R_{2 A}} N_{\theta_{A}}=1323$

e $N_{\text {bas }}=8424 * N_{v}$

${ }^{f}$ Cutoff energy $E_{\text {cut }}$ in $\mathrm{cm}^{-1}$, used to determine $N_{v}$. 
TABLE IV: Same as Table III but the monomer-fixed frame attached to $\mathrm{H}_{2} \mathrm{O}$ is an Eckart frame.

\begin{tabular}{lllrrrr}
\hline \hline state & Ref. 70 & DVR & \multicolumn{4}{c}{$|v\rangle$ basis } \\
\cline { 4 - 7 } & & $N_{\eta}=1323$ & $N_{v}=10$ & $N_{v}=30$ & $N_{v}=70$ & $N_{v}=100$ \\
& & & 6750. & 10600. & 15300. & 17690. \\
\hline \hline GS & 5023.765 & 0.000 & 8.252 & 0.149 & 0.002 & 0.000 \\
$v_{1}$ & 196.807 & 0.000 & -0.512 & -0.005 & 0.000 & 0.000 \\
$v_{2}$ & 332.103 & 0.000 & -0.752 & 0.080 & 0.002 & 0.000 \\
$2 v_{1}$ & 387.813 & 0.000 & -0.949 & -0.007 & 0.000 & 0.000 \\
$v_{1}+v_{2}$ & 519.606 & 0.000 & -1.275 & 0.073 & 0.002 & 0.000 \\
$2 v_{2}$ & 571.955 & 0.000 & -1.760 & 0.065 & 0.002 & 0.000 \\
$3 v_{1}$ & 573.258 & 0.000 & -1.451 & 0.002 & 0.001 & 0.000 \\
$2 v_{1}+v_{2}$ & 700.583 & 0.000 & -1.721 & 0.066 & 0.002 & 0.000 \\
$4 v_{1}$ & 751.907 & 0.000 & -1.636 & 0.022 & 0.002 & 0.000 \\
$v_{1}+2 v_{2}$ & 754.384 & 0.000 & -2.265 & 0.037 & 0.001 & 0.000 \\
$3 v_{2}$ & 854.914 & 0.000 & 3.022 & 0.305 & 0.008 & 0.001 \\
$3 v_{1}+v_{2}$ & 875.143 & 0.000 & -2.099 & 0.060 & 0.003 & 0.000 \\
$5 v_{1}$ & 925.563 & 0.000 & -1.834 & 0.016 & 0.002 & 0.000 \\
$2 v_{1}+2 v_{2}$ & 931.216 & 0.000 & -2.651 & 0.040 & 0.002 & 0.000 \\
\hline \hline
\end{tabular}


TABLE V: Action of inversion operator on the coordinates for three different cases

\begin{tabular}{|c|c|c|c|}
\hline$E$ & $E^{*}($ Case I $)$ & $E^{*}($ Case II $)$ & $E^{*}($ Case III $)$ \\
\hline$\alpha$ & $\pi+\alpha$ & $\pi+\alpha$ & $\pi+\alpha$ \\
\hline$\beta$ & $\pi-\beta$ & $\pi-\beta$ & $\pi-\beta$ \\
\hline$\alpha_{A}$ & $\pi-\alpha_{A}$ & $\pi-\alpha_{A}$ & $-\alpha_{A}$ \\
\hline$\beta_{A}$ & $\beta_{A}$ & $\beta_{A}$ & $\pi-\beta_{A}$ \\
\hline$\gamma_{A}$ & $\pi-\gamma_{A}$ & $-\gamma_{A}$ & $\gamma_{A}$ \\
\hline$\left(x_{i}, y_{i}, z_{i}\right)$ & $\left(-x_{i}, y_{i}, z_{i}\right)$ & $\left(x_{i},-y_{i}, z_{i}\right)$ & $\left(x_{i}, y_{i},-z_{i}\right)$ \\
\hline$\overline{|\xi\rangle^{a}}$ & $(-1)^{J}|\bar{\xi}\rangle^{b}$ & $(-1)^{J+k_{A}+k_{B}}|\bar{\xi}\rangle^{b}$ & $\left.\overline{(-1)^{J+j_{A}+j_{B}+k_{A}+k_{B}} \mid \bar{\xi}^{\prime}}\right\rangle$ \\
\hline
\end{tabular}



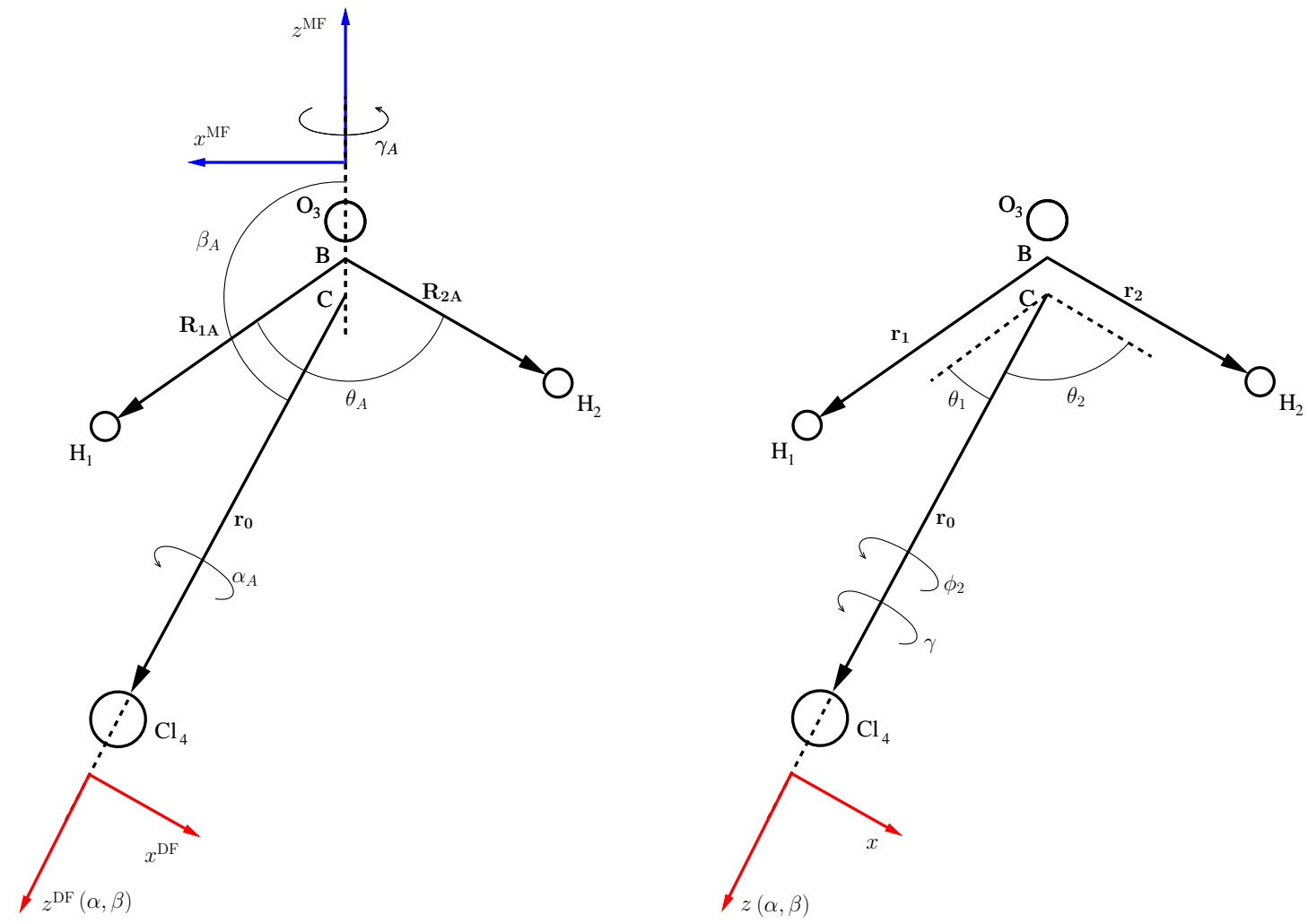

FIG. 1: Two sets of coordinates used to compute rovibrational levels of $\mathrm{H}_{2} \mathrm{O}_{-} \mathrm{Cl}^{-}$. The standard polyspherical coordinates $(\mathrm{B}),\left(\alpha, \beta, \gamma ; \theta_{1}, \theta_{2}, \phi_{2}, r_{0}, r_{1}, r_{2}\right)$ where a BF frame (only one frame) (obtained from the red frame of B by a third Euler angle rotation $\gamma$ ) is used to described the rotation of the molecule, The BAST coordinates (A), $\left(\alpha, \beta ; \alpha^{A}, \beta^{A}, \gamma^{A} ; r_{0}, R_{1 A}, R_{2 A}, \theta_{A}\right)$, which use Euler angles to describe the orientation of a MF frame (blue) with respect to the DF frame (red). Note $R_{1 A}=r_{1}$ and $R_{2 A}=r_{2}$ and therefore the stretch coordinates of the two sets of coordinates are identical. $\mathbf{R}_{\mathbf{1 A}}=\mathbf{r}_{\mathbf{1}}$ and $\mathbf{R}_{\mathbf{2} \mathbf{A}}=\mathbf{r}_{\mathbf{2}}$ are the Radau vectors of $\mathrm{H}_{2} \mathrm{O}$ ( $\mathrm{B}$ is the Radau canonical point, $\mathrm{C}$ is the centre of $\mathrm{H}_{2} \mathrm{O}$ ). Panel $\mathrm{B}$, for the polyspherical coordinates, is taken from X.-G. Wang and T. Carrington, Jr., J. Chem. Phys. 140, 204306 (2014), with the permission of AIP Publishing. 